\title{
Les méthodes de maîtrise de la reproduction disponibles chez les mammifères d'élevage et leur intérêt en agriculture biologique
}

\author{
M.-T. PELLICER-RUBIO'1, 2, 3, 4, S. FERCHAUD 5 , S. FRERET1, 2, 3, 4, H. TOURNADRE 6, A. FATET 1, 2, 3, 4 , \\ S. BOULOT ${ }^{7}$, J. PAVIE ${ }^{8}$, B. LEBOEUF ${ }^{5}$, F. BOCQUIER $9,10,11$ \\ 1 INRA, UMR85 Physiologie de la Reproduction et des Comportements, F-37380 Nouzilly, France \\ ${ }^{2}$ CNRS, UMR6175 Physiologie de la Reproduction et des Comportements, F-37380 Nouzilly, France \\ 3 Université François Rabelais de Tours, F-37041 Tours, France \\ ${ }^{4}$ Haras Nationaux, F-37380 Nouzilly, France \\ 5 INRA, UE88 Insémination caprine et porcine, F-86480 Rouillé, France \\ 6 INRA, UR1213 Herbivores, F-63122 Saint-Genès Champanelle, France \\ ${ }^{7}$ IFIP Institut du porc, F-35650 Le Rheu, France \\ 8 Institut de l'Elevage, Chambre Régionale d'Agriculture de Normandie, F-14053 Caen, France \\ ${ }^{9}$ INRA,UMR868 Élevage des Ruminants en Régions chaudes, F-34060 Montpellier, France \\ 10 Montpellier SupAgro, F-34060 Montpellier, France \\ 11 CIRAD, F-34060 Montpellier, France \\ Courriel : pellicer@tours.inra.fr
}

\begin{abstract}
La maîtrise de la reproduction, quelle que soit l'espèce concernée, est une pratique nécessaire en élevage. Elle met en œuvre des stratégies techniques ou de conduite des troupeaux permettant d'optimiser la reproduction (monte naturelle ou Insémination Artificielle (IA)) notamment en période d'anoestrus (saisonnier, post-partum), et ceci de façon adaptée aux différentes espèces dans leurs systèmes d'élevage (intensifs, extensifs).
\end{abstract}

Les stratégies de maîtrise de la reproduction mises en place par les éleveurs visent le choix de la période de mises bas, la synchronisation des mises bas, la diminution des périodes improductives (avancement/synchronisation de la puberté, reproduction à contre-saison, diminution de la durée de l'anoestrus post-partum), l'optimisation de la taille de la portée et enfin, l'application de l'insémination artificielle avec tous les avantages génétiques et sanitaires que celle-ci apporte (Chemineau et al 1996a, Fatet et al 2008). Les stratégies techniques ou de conduite utilisées aujourd'hui par les éleveurs pour la maîtrise de la reproduction concernent fondamentalement les traitements hormonaux d'induction et de synchronisa- tion de l'oestrus et de l'ovulation associés ou non à l'insémination artificielle, les traitements hormonaux pour déclencher les mises bas, les traitements photopériodiques de désaisonnement, l'effet mâle et des stratégies de conduite comme la conduite de la reproduction en bandes. Il y a des cas, beaucoup moins fréquents, où les éleveurs sont également concernés par la mise en œuvre de biotechnologies de la reproduction (transplantation embryonnaire, insémination intra-utérine). Le choix d'une stratégie ou d'une autre, ou bien d'une combinaison de celles-ci, va dépendre des spécificités de la physiologie de la reproduction de l'espèce que l'on considère (ovins, caprins, porcins ou bovins), du type de production (lait ou viande), du système de l'élevage, et également des objectifs des éleveurs en termes de productivité, d'adaptation au marché ou à la demande, ou d'intégration d'un mode de production spécifique à une Appellation d'Origine Contrôlée (AOC) ou à un label, comme par exemple le label de l'Agriculture Biologique (AB).

Cet article a comme objectifs de rappeler, espèce par espèce, les principes et objectifs des différentes stratégies de maîtrise de la reproduction mis en place dans des élevages conventionnels et de mener ensuite une discussion sur leurs avantages, leurs inconvénients et leurs limites dans le contexte spécifique de la production en $\mathrm{AB}$.

Le règlement CEE $n^{\circ}$ 2092/91 sur le mode de production des animaux en $\mathrm{AB}$ a été abrogé et remplacé à partir du 1 $\mathrm{er}$ Janvier 2009 par le nouveau règlement européen CE n 834/2007 et ses règles d'application $\mathrm{CE} \mathrm{n}^{\circ} 889 / 2008$ $(05 / 09 / 2008)$. Ces nouveaux textes modifient un certain nombre de points relatifs aux conditions de production. Les points de discussion de cet article prendront en compte les conséquences de cette nouvelle réglementation sur les stratégies de choix pour la maîtrise de la reproduction des animaux en $\mathrm{AB}$.

\section{1 / Maîtrise de la reproduc- tion chez les ovins et caprins}

La reproduction chez les ovins et caprins est saisonnée. Chez les deux sexes, il existe au cours de l'année une période d'activité sexuelle maximale 
(saison sexuelle), en général d'août à janvier, et une autre d'activité minimale ou de repos sexuel (contre-saison), en général de février à juillet. La durée de la saison sexuelle est très variable selon les races. Pendant la saison sexuelle, les femelles non fécondées présentent des cycles ovariens réguliers d'environ $17 \mathrm{j}$ (brebis) ou $21 \mathrm{j}$ (chèvre). La période de repos sexuel chez la femelle se caractérise par l'absence d'oestrus, le plus souvent associée à l'absence d'ovulation (anoestrus saisonnier). Chez le mâle, on observe en contre-saison une diminution des niveaux de testostérone, du volume testiculaire, du comportement sexuel et de la production quantitative et qualitative de spermatozoïdes (Chemineau et al 1992, Baril et al 1993). La durée moyenne de gestation est comprise entre 144 et $152 \mathrm{j}$ dans les deux espèces. Dans l'espèce ovine la taille moyenne de la portée varie selon les races entre 1 et 3,5 agneaux par mise bas. La plupart de races de chèvres françaises ont une prolificité de 1,5 à 2,5 chevreaux par mise bas (Baril et al 1993).

\section{1 / En élevage ovin et caprin conventionnel}

Environ 50 races ovines à viande, d'effectifs très différents, sont recensées en France. En élevage ovin allaitant, la conduite de reproduction la plus fréquente est basée sur une mise bas par brebis et par an. Dans ces conditions, nous pouvons observer une seule période annuelle de mises bas par troupeau ou - cas le plus courant - par lot. Cependant, pour améliorer la productivité numérique des brebis, il est possible d'augmenter le nombre de portées par an ( 3 agnelages en 2 ans ou 4 agnelages en 3 ans), ce qui nécessite la mise à la reproduction à différentes périodes de l'année (Bonnes et al 2005). La productivité numérique peut aussi être améliorée en augmentant la taille de la portée : par le choix de races prolifiques, la pratique d'un flushing en période de reproduction ou l'utilisation de traitements hormonaux (Chemineau et al 1996a).

Les troupeaux ovins laitiers en France sont principalement localisés dans le rayon de Roquefort (race Lacaune), les Pyrénées-Atlantiques (races Manech, Basco-Béarnaise) et la Corse (race Corse). La conduite de reproduction est caractérisée par une mise bas par campagne avec un saisonnement marqué à très marqué selon les régions. La mise à la reproduction a lieu à contre-saison en fin de printemps et début d'été pour caler le début de la lactation avec la date d'ouverture des laiteries (de décembre à août). La durée de la lactation est d'environ 8 mois (Bonnes et al 2005).

Les races Saanen et Alpine sont les principales races caprines laitières françaises. Le cycle de production d'une chèvre laitière est de 12 mois $(10$ mois de lactation dont 3 mois de gestation en fin de lactation, suivis de 2 mois de tarissement en fin de gestation). Ceci implique qu'une femelle n'est mise à la reproduction qu'une seule fois par an. L'éleveur peut alors choisir une seule période de mises bas par an pour l'ensemble du troupeau (cas le plus fréquent), ou bien travailler avec plusieurs lots de mises bas (Bonnes et al 2005).

Ainsi, les stratégies de maîtrise de la reproduction mises en place par les éleveurs ovins et caprins ont comme objectifs principaux (Chemineau et al 1996a) :

- l'obtention d'une plus grande souplesse dans le choix des périodes des mises bas et des livraisons de lait ou d'agneaux, grâce à la maîtrise de la saisonnalité ;

- la réduction des périodes improductives par la maîtrise de la saisonnalité, mais aussi par l'avancement de l'âge à la première saillie et la réduction de la durée de l'anœstrus post-partum ;

- le groupage des mises bas par la synchronisation des fécondations, ce qui facilite l'organisation du travail, permet une meilleure surveillance des naissances et simplifie la gestion du troupeau de par la constitution de lots assez homogènes ;

\section{- le progrès génétique via l'IA.}

Pour atteindre ces objectifs les éleveurs disposent des différentes techniques : les traitements hormonaux, l'effet mâle et les traitements photopériodiques. De plus, il existe des approches de conduite en production de lait de chèvre (les mises bas tardives et les lactations longues) permettant également de contrer les effets de la saisonnalité.

a) Traitements hormonaux pour l'induction et la synchronisation des chaleurs et des ovulations

Le traitement hormonal d'induction et de synchronisation de l'oestrus et de l'ovulation est la technique de choix pour l'application de l'IA. En effet, la synchronisation hormonale des chaleurs est associée à l'IA dans plus de
$80 \%$ des cas. En dehors du cadre de l'IA, ces traitements sont utilisés comme technique de désaisonnement et/ou pour obtenir un bon groupage de mises bas.

Les traitements utilisés en France par les éleveurs consistent à administrer un progestagène de synthèse (éponge vaginale imprégnée d'acétate de fluorogestone : FGA) suivi par une injection d'eCG (choriogonadotropine équine). En outre, chez la chèvre, du cloprosténol (un analogue de synthèse de la prostaglandine $\mathrm{F} 2 \alpha$ ) est aussi co-administré avec l'eCG. Ces traitements induisent une ovulation environ $66 \mathrm{~h}$, chez la brebis, et $55 \mathrm{~h}$, chez la chèvre, après le retrait des éponges (Baril et al 1993).

Ces traitements hormonaux sont très efficaces à n'importe quelle période de l'anoestrus saisonnier, aussi bien qu'en saison sexuelle et quel que soit le stade physiologique de la femelle hors gestation. Ils assurent une très bonne synchronisation des ovulations, de l'ordre de 12-24 h, et par conséquent une très bonne synchronisation des mises bas après fécondation par monte naturelle ou par insémination artificielle. Dans le cas de l'IA, la très forte synchronisation des ovulations induites permet d'obtenir des résultats de fertilité satisfaisants après une seule IA à un moment prédéterminé et sans détection des chaleurs : $55 \mathrm{~h}$ après le retrait de l'éponge chez la brebis, $43 \mathrm{~h}$ à $45 \mathrm{~h}$ après retrait chez la chèvre. De ce fait, la synchronisation hormonale des ovulations a favorisé le développement de l'IA et augmenté l'efficacité des schémas de sélection (David et al 2008, Fatet et al 2008).

Aujourd'hui, chez les ovins et caprins, plus de $99 \%$ des IA sont réalisées après induction et synchronisation hormonale des ovulations. Cette pratique est largement utilisée en brebis laitière ( $42 \%$ du cheptel national). En ovin allaitant, l'IA est peu utilisée (4\% du cheptel national). L'effectif inséminé est également faible $(9 \%)$ chez les caprins (tableau 1). L'activité d'IA est très saisonnée dans les deux espèces avec un clair intérêt de l'IA pour la reproduction à contre-saison ou en avance de saison sexuelle. En brebis laitière, $89 \%$ des IA sont réalisées de mai à septembre avec un pic important au mois de juin En ovin allaitant, il n'y a pas de pic aussi marqué mais $79 \%$ des IA sont réalisées de mars à juillet. Chez les caprins, $82 \%$ de l'activité se place entre mars et août dont $36 \%$ en avriljuin (Fatet et al 2008). 
Tableau 1. Les schémas de sélection en France (Fatet et al 2008).

\begin{tabular}{|l|c|c|c|}
\hline & $\begin{array}{c}\text { Ovins } \\
\text { laitiers }\end{array}$ & $\begin{array}{c}\text { Ovins } \\
\text { allaitants }\end{array}$ & Caprins \\
\hline Cheptel national & 1492000 & 4869000 & 850000 \\
Effectif en contrôle performance/laitier & 302000 & 357000 & 300000 \\
Races ayant un schéma de sélection & 5 & 21 & 2 \\
Effectif inséminé & 625000 & 216000 & 79000 \\
$\%$ du cheptel inséminé & 41,9 & 4,4 & 9,2 \\
\hline
\end{tabular}

Le principal problème pratique lié à l'utilisation de traitements hormonaux vient de la réponse immunitaire à l'eCG des brebis et des chèvres. La production d'anticorps anti-eCG se traduit par une baisse de fertilité après IA à temps fixe du fait d'un retard dans l'apparition des ovulations (Roy et al 1999). Pour cette raison, il est conseillé chez les caprins de limiter à 3 le nombre de traitements au cours de la carrière d'une femelle. En ovin lait et viande, des outils d'aide à la décision sont mis en place sur le terrain. Le conseil n'est pas raisonné en terme de limitation du nombre de traitements mais d'analyse de la carrière reproductive de la brebis (par exemple, les brebis n'ayant pas été fertiles à l'IA l'année précédente peuvent être écartées des campagnes d'IA suivantes). Cependant, les conséquences liées aux anticorps anti-eCG sont négligeables lorsque le traitement hormonal est suivi d'une saillie naturelle. Enfin, il faut rappeler que l'induction hormonale des ovulations ne permet pas d'induire une activité ovulatoire cyclique soutenue en contre-saison. En revanche, celle-ci peut être obtenue si l'on associe des traitements lumineux de désaisonnement. Il ne faut pas non plus oublier que le succès de la reproduction suite à un traitement hormonal des femelles nécessite aussi de disposer de semence de qualité. A contre-saison celle-ci peut être obtenue soit en utilisant des mâles naturellement peu saisonnés ou désaisonnés par traitement photopériodique, soit en utilisant de la semence congelée en pleine saison sexuelle.

\section{b) L'effet mâle}

L'effet mâle est une technique permettant d'induire des ovulations synchrones en dehors de la saison sexuelle chez des femelles anovulatoires. L'effet mâle seul ne permet pas la synchronisation des ovulations chez des femelles cycliques et par conséquent il n'est donc pas efficace en saison sexuelle. Cette technique constitue cependant une alternative à l'utilisation d'hormone pour obtenir un bon groupage de mises bas à faible coût hors période sexuelle. Il s'agit aussi d'une pratique prometteuse pour mettre en place l'IA.

L'efficacité de la réponse à l'effet mâle dépend d'un grand nombre de facteurs : race, moment de l'anoestrus, âge, nutrition, condition d'élevage, etc. (Walkden-Brown et al 1999, Thimonier et al 2000). Dans le cas des races très saisonnées, son efficacité se voit souvent restreinte à la période proche du début de la saison sexuelle pour avancer la saison de reproduction mais elle est trop peu efficace pour induire une reproduction à contre-saison. Dans cette situation, le traitement des femelles et/ou des mâles avec la photopériode est un moyen complémentaire pour optimiser la réponse à l'effet mâle tout au long de l'anoestrus saisonnier (Chemineau et al 1986, 1996b, Flores et al 2000, Thimonier et al 2000).

Une réponse efficace à l'effet mâle, chez la chèvre et la brebis en anoestrus anovulatoire, est caractérisée par l'induction d'une ovulation dans les 3 premiers jours qui suivent l'introduction du mâle. La première ovulation induite par effet mâle est suivie soit d'une phase lutéale de durée normale (cycle normal) soit d'une phase lutéale de courte durée due à une lutéolyse précoce (cycle court). Ce cycle court est normalement suivi, 5-6 j après, par un cycle normal. Ensuite, plusieurs cycles normaux peuvent se succéder dans le cas de l'installation d'une activité ovulatoire cyclique (Chemineau 1989, Walkden-Brown et al 1999).

L'ovulation correspondant aux cycles courts est toujours silencieuse chez les ovins, mais peut être accompagnée de comportement d'oestrus chez certaines chèvres. L'ovulation correspondant au premier cycle normal, précédé ou non d'un cycle court, est accompagnée de comportement d'oestrus chez les caprins. Cette ovulation est aussi silencieuse chez les ovins. Les cycles normaux suivants sont accompagnés de comportement d'oestrus dans les deux espèces (Walkden-Brown et al 1999).
Dans un troupeau, le fait que certaines femelles développent un cycle court et d'autres un cycle normal, induit deux pics de mises bas correspondant à deux pics de fécondation après l'introduction des mâles (les 3 ème et 8 ème $j$ environ chez les caprins

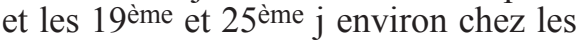
ovins).

Actuellement, dans les deux espèces, le seul moyen d'induire de façon systématique un cycle normal par effet mâle consiste à réaliser un traitement progestatif (progestérone ou progestagène de synthèse) des femelles avant l'introduction du mâle (Chemineau 1989, Walkden-Brown et al 1999, Thimonier et al 2000).

La synchronisation de la première ovulation fertile induite par effet mâle est moins marquée qu'après traitement hormonal. De plus, pour synchroniser au mieux les ovulations, l'effet mâle doit être réalisé à une période de l'anoestrus pendant laquelle la proportion de femelles cycliques est très faible (figure 1). L'effet mâle est en général suivi d'une monte naturelle. Il est toutefois possible de pratiquer l'IA sur chaleurs naturelles (Corke 1982, Restall 1988), mais ces protocoles sont très peu utilisés en France car les IA d'un même lot s'étalent alors sur plusieurs jours.

Le traitement hormonal des chèvres avec des progestatifs et/ou des prostaglandines augmente la synchronie des ovulations fertiles induites par effet mâle (de l'ordre de 24 h, figure 2) et permet une seule insémination à un moment fixe sans détection de chaleurs, avec des résultats de fertilité similaires à ceux obtenus avec le traitement hormonal classique (LópezSebastian et al 2007, Pellicer-Rubio et al 2008). Ces nouveaux protocoles commencent à être utilisés sur le terrain et représentent une excellente alternative à l'utilisation d'eCG. Les modalités d'utilisation de l'IA avec ce type de protocoles n'ont pas encore été étudiées chez les ovins.

\section{c) Traitements photopériodiques}

Les changements graduels de la durée du jour (photopériode) au cours de l'année contrôlent les variations saisonnières de la reproduction des ovins et caprins. La mélatonine produite par la glande pinéale est 1'hormone qui permet à l'animal de mesurer la durée du jour (Chemineau et Malpaux 1998). 
Figure 1. Distribution de mises bas en lutte naturelle après effet mâle en race Limousine (lutte pendant $45 \mathrm{j}$ ).

Cas d'un troupeau avec une forte ou une faible proportion de brebis cycliques avant l'introduction des béliers (64\%, $n=100$ vs 13\%, $n=70)$. Toutes les brebis non cycliques ont ovulé en réponse à l'effet mâle. La fertilité globale est de $86 \%$ (lutte en avril, lot avec $64 \%$ de brebis cycliques) et de $94 \%$ (lutte en juin, lot avec $13 \%$ de brebis cycliques), respectivement (Plate-forme de Recherche en AB de I'INRA de ClermontTheix : Tournadre et al 2005, 2008, résultats non publiés).

\section{Nombre de brebis mettant bas}
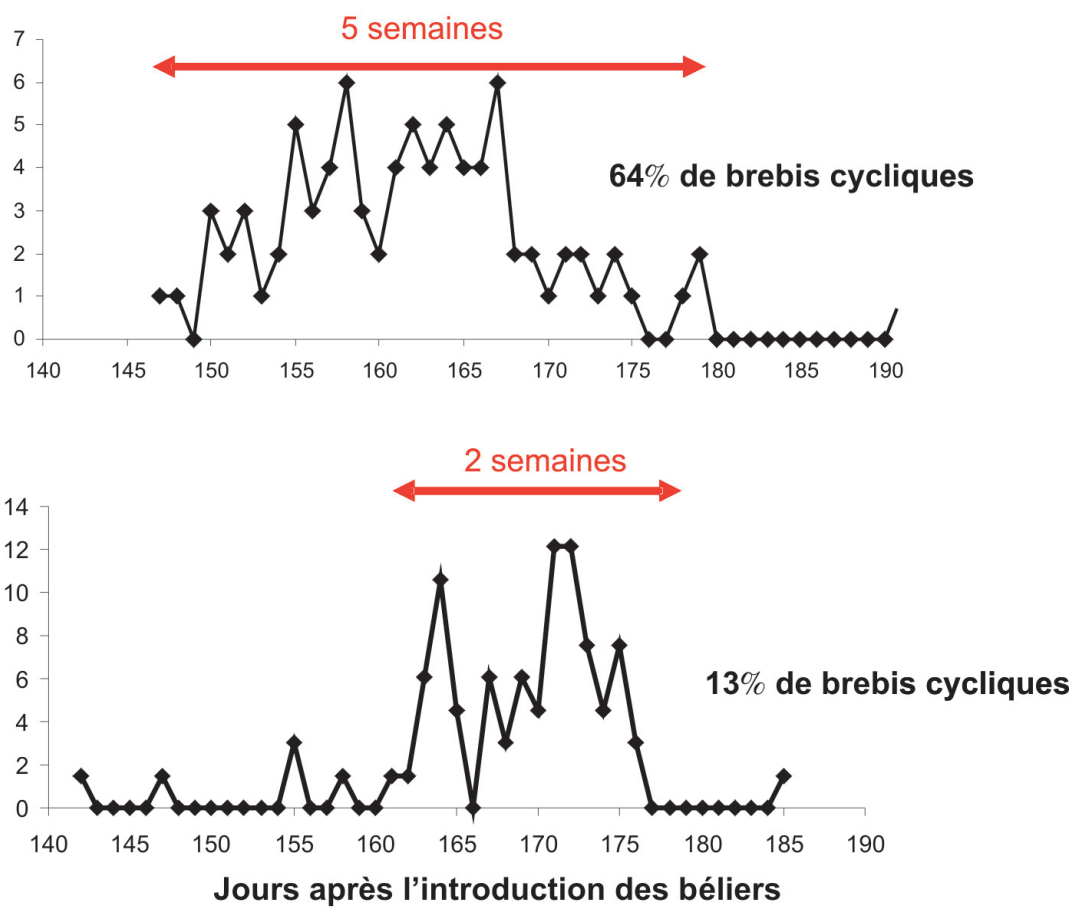

Figure 2. Synchronisation du pic preovulatoire de LH correspondant au premier cycle normal induit par effet mâle, précédé ou non d'un cycle court, chez la chèvre laitière.

Chez des chèvres Saânen et Alpine traitées avec des progestatifs (éponges vaginales de FGA) pendant les 11 j qui précèdent l'introduction des mâles, la première ovulation induite par le mâle (ovul 1) est accompagné de comportement d'oestrus et suivi d'une phase lutéale normale. La plupart des chèvres manifestent le pic preovulatoire de LH 33-56 h après l'introduction des boucs (synchronisation de 24 h) (Pellicer-Rubio et al 2008). L'utilisation de l'effet mâle sans hormones induit chez la plupart de chèvres un premier cycle court puis une deuxième ovulation (ovul 2) accompagnée de comportement d'oestrus et suivi d'une phase lutéale normale. Le pic preovulatoire de LH de cette deuxième ovulation apparaît entre $\mathrm{J} 6$ et $\mathrm{J} 9$ après l'introduction des boucs (synchronisation de 48 h) (Pellicer-Rubio et al 2007, résultats non publiés).

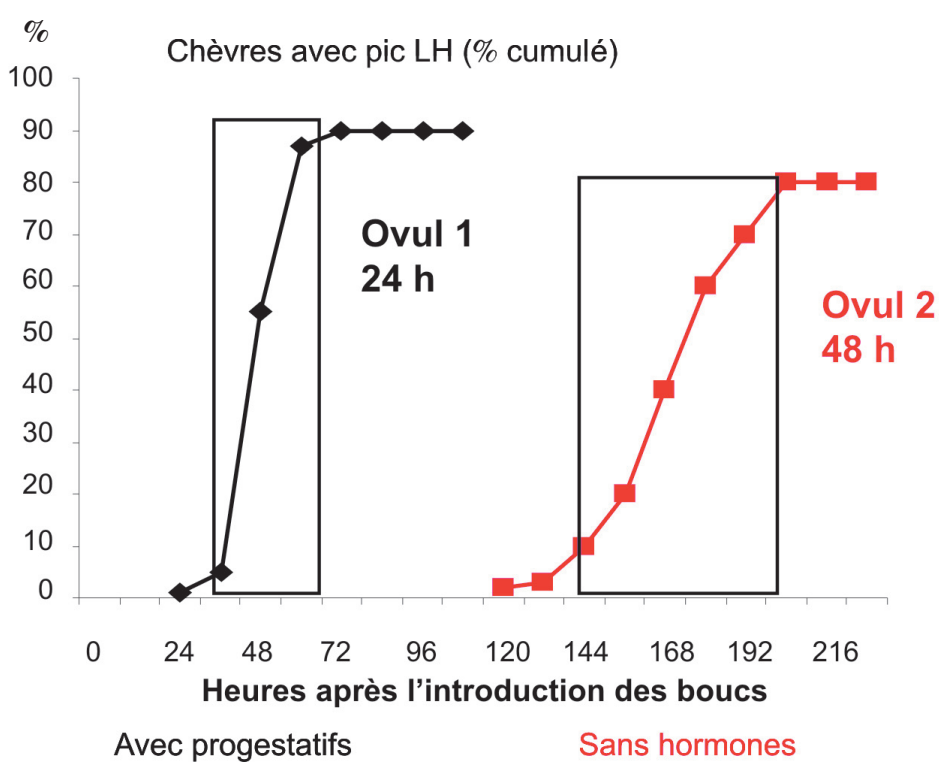

La manipulation de la photopériode permet de maîtriser la saisonnalité et de rendre possible la reproduction en dehors de la saison sexuelle. Les traitements photopériodiques sont basés sur la perception par les animaux d'une alternance de Jours Longs (JL : plus de $12 \mathrm{~h}$ de lumière par jour suite à des jours courts) et de Jours Courts (JC : moins de $12 \mathrm{~h}$ de lumière par jour suite à des jours longs), alternance qui existe en photopériode naturelle. Classiquement, un traitement de JL comprend des jours de $16 \mathrm{~h}$ de lumière par $24 \mathrm{~h}$, et un traitement de JC comprend des jours de $8 \mathrm{~h}$ de lumière par $24 \mathrm{~h}$, ce qui correspond à la durée du jour au solstice d'été ou d'hiver, respectivement, sous nos latitudes. Les JL sont inhibiteurs de l'activité sexuelle, alors que les jours courts sont stimulateurs (Chemineau et al 1992).

En pratique, les JL et les JC peuvent être facilement appliqués dans des bâtiments étanches à la lumière, puisqu'il suffit de contrôler la durée d'éclairement journalière des animaux, mais de telles installations sont coûteuses. Dans des bâtiments ouverts (cas général dans les élevages), les JL sont administrés en éclairant les animaux avec de la lumière artificielle, ou bien en profitant des jours longs naturels. Les JC sont obtenus soit en procurant l'obscurité totale pendant $16 \mathrm{~h}$ ( $\mathrm{JC}$ artificiels, difficile à réaliser en ferme), soit en profitant de jours courts naturels ou, plus fréquemment, en posant par voie sous-cutanée des implants de mélatonine dont l'effet est équivalent à celui des jours courts (Chemineau et al 1992).

La principale contrainte liée à l'application de JC artificiels se trouve dans la difficulté de créer l'obscurité totale pour obtenir $16 \mathrm{~h}$ de nuit à des moments de l'année où naturellement la durée journalière d'éclairement est supérieure à $12 \mathrm{~h}$. De plus, il est impératif de ne pas éclairer, même momentanément, pendant les heures d'obscurité et par conséquent, aucune intervention des éleveurs ne peut être réalisé pendant la phase d'obscurité. Par conséquent, les horaires d'obscurité ne sont toujours pas compatibles avec les heures de traite et/ou chez des animaux sortant au pâturage. Devant ces contraintes, la plupart des éleveurs en conventionnel préfèrent remplacer les JC artificiels par les implants de mélatonine (Brice 2003).

Aujourd'hui, les éleveurs utilisent différents traitements photopériodiques de désaisonnement leur permettant la 
Figure 3. Traitements photopériodiques en bâtiments ouverts.

a) Un traitement lumineux de jours longs artificiels est administré pendant 2-3 mois au cours de l'hiver. Ce traitement doit finir au plus tard fin mars. Ensuite les animaux reçoivent la photopériode naturelle. Les femelles sont mises en lutte environ 35-45 j, chez la brebis, ou 50-70 j, chez la chèvre, après l'arrêt du traitement lumineux afin de profiter d'un effet mâle.

b) Un traitement lumineux de jours longs artificiels est administré pendant 2-3 mois puis arrêté au cours des mois d'avril-mai. Ensuite les animaux reçoivent des implants de mélatonine. Les femelles sont mises en lutte environ 35-45 j, chez la brebis, ou $50-70$ j, chez la chèvre, après l'arrêt du traitement lumineux afin de profiter d'un effet mâle.

c) Les animaux sont maintenus en photopériode naturelle et à partir du mois de juin ils reçoivent des implants de mélatonine. Les femelles sont mises en lutte environ 35-45 j, chez la brebis, ou 50-70 j, chez la chèvre, après le jour de la pose des implants afin de profiter d'un effet mâle. En AB, la mélatonine doit être remplacée par un traitement de JC artificiels. La durée du traitement de JC est d'environ 3 mois (arrêt à la fin de la lutte). Quel que soit le traitement photopériodique, il est conseillé de traiter mâles et femelles pour optimiser les résultas de fécondité.

a $\quad$ Lutte

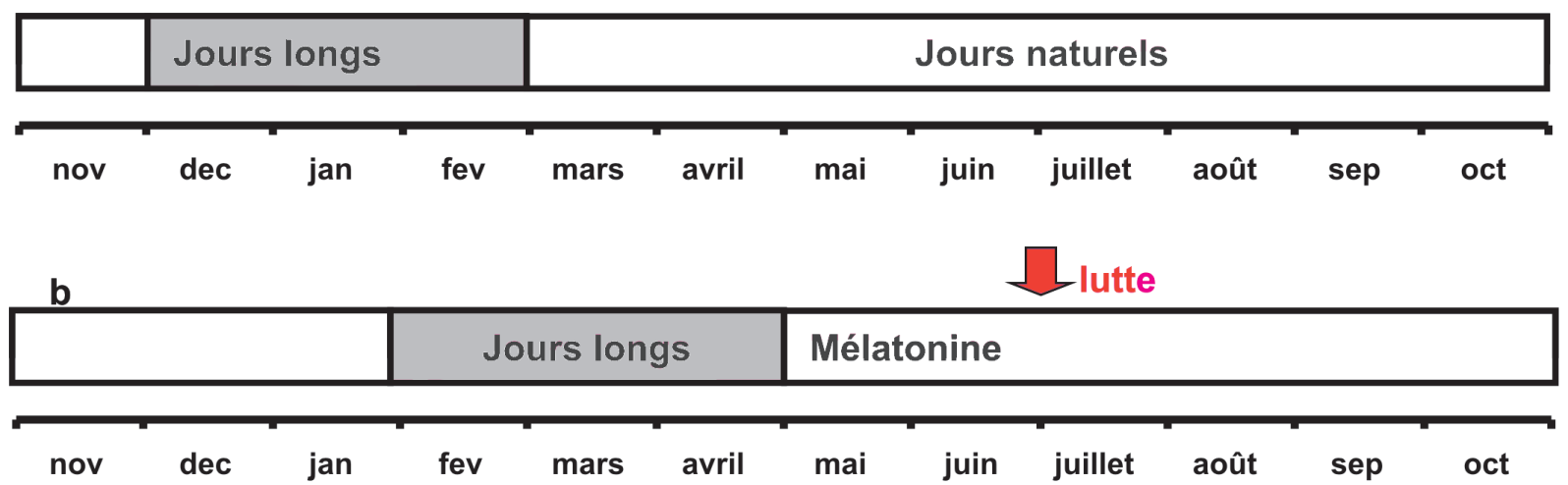

c Dlutte

\begin{tabular}{|c|c|l}
\hline$c$ & Mélatonine \\
\hline nov dec jan fev mars avril mai juin juillet août sep oct
\end{tabular}

mise à la reproduction tout au long de l'anoestrus saisonnier (figure 3) :

- pour des mises à la reproduction au printemps (avril-mai), un traitement lumineux de jours longs artificiels est administré au cours de l'hiver. Ce traitement doit finir au plus tard fin mars (équinoxe de printemps). Ensuite les animaux reçoivent la photopériode naturelle, encore de courte durée sous nos latitudes ce qui stimule l'activité sexuelle (figure 3a). Dans ces conditions, les jours naturels qui succèdent aux JL sont aussi efficaces que les JC artificiels ou que la mélatonine pour stimuler l'activité sexuelle. Ce traitement s'est avéré être très efficace chez les caprins des races laitières françaises (Chemineau et al 1999, Pellicer-Rubio et al 2007). Chez les ovins, l'efficacité de ce traitement a également été démontrée chez le bélier (figure 4) ;

- pour des mises à la reproduction plus tardives (juin-juillet) (figure $3 b$ ), un traitement lumineux de JL est aussi administré puis arrêté au cours des mois d'avril-mai. A ce moment là, les jours naturels ne sont pas suffisamment courts par rapport au traitement de JL. Pour cette raison, il est nécessaire d'utiliser des JC artificiels ou d'administrer des implants de mélatonine pour stimuler l'activité sexuelle (Chemineau et al 1992) ;

- enfin, dans l'objectif d'avancer la date du début de la saison sexuelle chez des races très saisonnées dont le début de la saison se situe en septembre-octobre, les animaux peuvent être maintenus en photopériode naturelle tout au long de l'hiver et du printemps et recevoir à partir du mois de juin des JC artificiels ou des implants de mélatonine (figure $3 \mathrm{c}$ ). Ce traitement permet d'avancer la saison sexuelle de 1 à 1,5 mois (Chemineau et al 1992, 1996b).

En France, les traitements photopériodiques sont surtout utilisés par les éleveurs caprins. Une enquête réalisée par l'Institut de l'Elevage en 2002 dans 48 départements ayant plus de 10 éleveurs caprins adhérant au contrôle laitier, montre que 27\% des 1995 élevages enquêtés pratiquaient le photopériodisme. L'application de JL artificiels suivis de jours naturels est le protocole le plus utilisé $(60 \%$ des élevages qui pratiquent le photopériodis- me). Les JL artificiels suivis de mélatonine, ou bien l'utilisation de mélatonine seule sont aussi des pratiques observées dans les élevages caprins (Brice 2003). L'utilisation d'implants de mélatonine pour avancer la période de reproduction est une technique également pratiquée en ovin viande, alors que les protocoles basés sur l'application de JL artificiels sont encore peu employés. En ovin laitier, le photopériodisme n'est pas utilisé en élevage.

Le principal avantage des traitements photopériodiques est celui d'induire l'activité sexuelle à contre-saison simultanément chez le mâle et la femelle. De plus, ils induisent une activité ovulatoire cyclique (3-4 cycles consécutifs) permettant aux femelles d'être fécondés aux retours de chaleurs dans le cas d'un échec de reproduction après une première mise à la reproduction. Cependant, cette technique ne permet pas de groupement de chaleurs, les ovulations induites s'échelonnent sur 2-3 semaines ce qui rend difficile la mise en place de l'IA. Le groupage des mises bas s'obtient en associant une méthode de synchronisation des 
Figure 4. Traitement photopériodique sans mélatonine pour des mises à la reproduction au printemps chez le bélier.

Evolution du poids testiculaire dans 3 lots de 8 béliers lle-de-France logés en bâtiment ouvert et qui reçoivent :

1) soit la photopériode naturelle (lot $\mathrm{JN}$ : témoin négatif), 2) soit un traitement de jours longs (16 h de lumière par jour de $6 \mathrm{~h}$ à $22 \mathrm{~h}$ ) suivi d'un traitement avec de la mélatonine (lot $\mathrm{JL}+$ mél : témoin positif), 3) soit un traitement de JL pour ensuite recevoir à nouveau les jours naturels (lot $\mathrm{JL}+\mathrm{JN}$ ). Suite au traitement de $\mathrm{JL}$, l'activité sexuelle des béliers (mesurée par le poids testiculaire) atteint son maximum 45-60 j après l'arrêt des $\mathrm{JL}$, à une période où le niveau d'activité sexuelle des béliers en photopériode naturelle est faible. Les JL s'étant arrêtés mi-février, les JN qui succèdent sont aussi efficaces que la mélatonine pour stimuler l'activité sexuelle au printemps. Ensuite, le poids testiculaire des mâles traités diminue progressivement alors que celui des mâles témoins en lumière naturelle augmente pour atteindre leur maximum en saison sexuelle (Pellicer-Rubio et al 2008, résultats non publiés).

\section{Jours longs Mélatonine ou jours naturels}

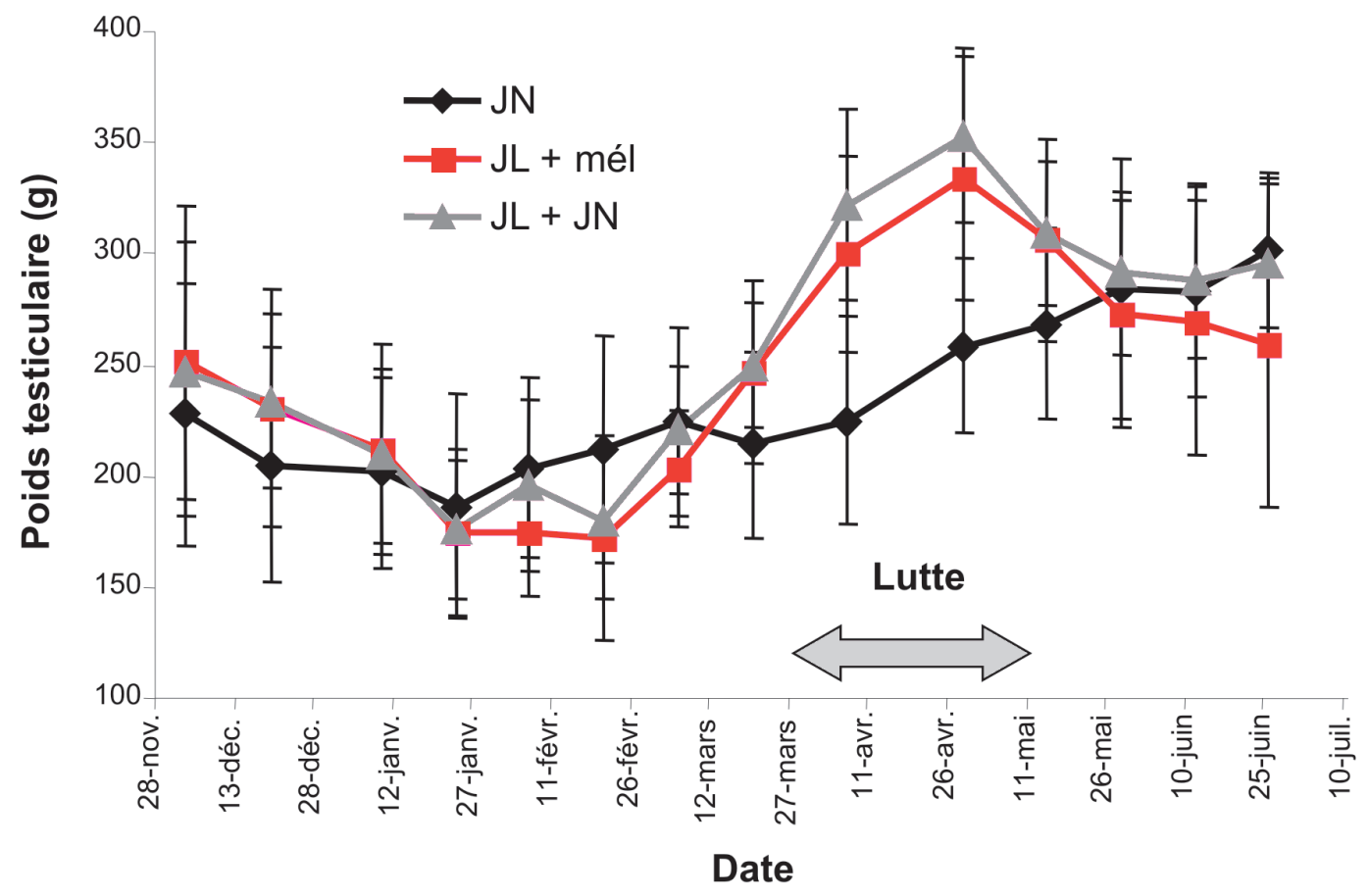

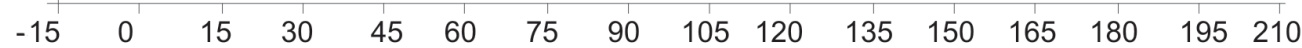

\section{Jours avant et après le démarrage des jours longs}

ovulations comme le traitement hormonal ou l'effet mâle. En monte naturelle, il est d'ailleurs conseillé d'associer un effet mâle pour augmenter les résultats de fertilité et de prolificité. La lutte devra alors commencer peu avant le démarrage de la cyclicité induite par le traitement photopériodique pour pouvoir bénéficier d'un effet mâle. Si les mâles sont introduits une fois que les femelles ont démarré une activité ovulatoire cyclique, ils pourront assurer les fécondations mais sans groupage des mises bas (Chemineau et al 1992, 1996b). Enfin, il faut rappeler qu'après la stimulation de l'activité sexuelle à contre-saison par les traitements photopériodiques, la re-synchronisation de l'activité de reproduction avec la photopériode naturelle n'est pas immédiate. Celle-ci s'installe progres- sivement, et l'activité sexuelle peut être perturbée durant la saison de reproduction naturelle qui succède (Delgadillo et al 2001). Cet effet à long terme n'a pas encore été bien caractérisé.

d) Les lactations longues et les mises bas tardives chez la chèvre laitière

Selon les objectifs des producteurs, des stratégies de conduite du troupeau peuvent également se mettre en place pour gérer la saisonnalité (Bossis et al 2008). Par exemple, le recours à des «lactations longues» (de plus de $300 \mathrm{j}$ ) constitue un outil intéressant de gestion de la reproduction en facilitant 1) le «recalage» des chèvres décalées sur le reste du troupeau (alternative à la réforme anticipée de chèvres en échec de reproduction, recalage de primipares, dont les mises bas sont habituellement plus tardives, avec le troupeau adulte), 2) le maintien dans le troupeau de bonnes chèvres laitières mais avec des problèmes de reproduction et 3) l'étalement de la production laitière. Cette technique est ainsi associée au désaisonnement. Elle apparaît parfois comme subie car orientée vers la gestion de chèvres ayant des problèmes de reproduction, mais peut néanmoins être aussi envisagée comme une technique à part entière en vue de la simplification de la conduite des troupeaux (Bossis et al 2008).

Les mises bas qualifiées de «tardives») (à partir de mars et jusqu'à la fin juin) représentent environ $30 \%$ des mises bas annuelles (23\% en mars et $7 \%$ d'avril à juin). Elles constituent une 
pratique d'élevage permettant de maximiser le lait d'automne pour bénéficier d'un prix du lait intéressant sans faire intervenir des traitements de désaisonnement du troupeau. Plus les mises bas sont tardives, plus l'intérêt économique sur le produit lait est important. Il s'agit d'un itinéraire alternatif pour gérer la saisonnalité. Cette pratique est très adaptée au pâturage et à l'affouragement à l'herbe et permet ainsi de valoriser les surfaces en herbe dans certaines régions en profitant pleinement de l'herbe au printemps. Elle constitue aussi un moyen de gérer d'éventuels échecs de reproduction (Institut de l'élevage et Office de l'élevage 2008).

\section{2 / En élevage ovin et caprin biologique}

La réglementation européenne de la production $\mathrm{AB}$ a des conséquences importantes pour le choix des stratégies de maîtrise de la reproduction à mettre en place. Les trois points principaux de la législation qu'il faut considérer sont les suivants :

- la reproduction en $\mathrm{AB}$ «recourt à des méthodes naturelles. Toutefois, l'insémination artificielle est autorisée. $D^{\prime}$ autres formes de reproduction artificielle telles que le clonage et le transfert d'embryons sont interdites». Le nouveau règlement ne spécifie pas la technique précise d'IA qui est autorisée, cependant il semble logique que seule l'IA par voie vaginale serait praticable en AB. En effet, l'IA par voie intra-utérine nécessite une intervention chirurgicale (laparoscopie), ce qui va à l'encontre des objectifs généraux en $\mathrm{AB}$;

- le règlement interdit explicitement «l'utilisation d'hormones ou autres substances analogues en vue de maîtriser la reproduction ou à d'autres fins (par exemple, induction ou synchronisation des chaleurs)». Ceci exclut donc le recours aux traitements hormonaux pour l'induction et la synchronisation des chaleurs et des ovulations, ainsi qu'à la mélatonine ;

- concernant les traitements lumineux, aucune mention n'est faite spécifiquement pour les petits ruminants, ni pour les mammifères domestiques en général. Nous pouvons alors considérer que ce sont les «normes minimales relatives à la protection des animaux dans les élevages» (directive communautaire 98/58/CE) qui s'appliquent. Ainsi, les dispositions générales concernant les bâtiments et locaux de stabulation soulignent clairement que "les animaux ne doivent pas être maintenus en permanence dans l'obscurité ni être exposés sans interruption appropriée à la lumière artificielle». Les traitements lumineux respectant ces normes minimales pourraient donc être pratiqués en $\mathrm{AB}$.

En pratique, l'effet mâle reste le seul moyen disponible en $\mathrm{AB}$ pour obtenir une bonne synchronisation de mises bas sans recours aux hormones. Par conséquent, en $\mathrm{AB}$ le groupage des mises bas ne peut être obtenu que dans les lots mis à la reproduction à contresaison ou en avance de saison. De plus, le traitement lumineux des femelles et/ou des mâles peut s'avérer nécessaire pour optimiser la réponse à l'effet mâle, selon le moment de l'anoestrus et le degré de saisonnalité des différentes races. Il convient aussi d'observer certaines règles d'application qui ont été vérifiées sur la race Limousine dans le cadre des travaux de la plate-forme de recherche en $\mathrm{AB}$ de l'INRA de Clermont-Theix (Tournadre et al 2002, 2008) :

- le moment de l'anoestrus pendant lequel la réponse à l'effet mâle est maximale dépend de la saisonnalité de la race (figure 5, expérience 1). En outre, plus la proportion de brebis cycliques est faible, meilleure sera la synchronisation des mises bas dans le troupeau (cf. figure 1) ;

- l'intervalle entre le tarissement et la mise en lutte doit être d'environ $80 \mathrm{j}$ pour améliorer la réponse ovulatoire à 1'effet mâle (figure 5, expérience 2) ;

- une supplémentation alimentaire (flushing) n'a pas d'effet sur l'efficacité de la réponse à l'effet mâle, au moins chez des animaux en bon état corporel (figure 5, expérience 3).

Le choix de la période de mises bas en $A B$ serait facilité par l'application de traitements lumineux pour optimiser la reproduction tout au long de l'anoestrus saisonnier, de fin mars à septembre. Les difficultés techniques liées à la mise en place des traitements lumineux sont similaires pour les élevages $\mathrm{AB}$ et conventionnels. Toutefois, ne pouvant pas utiliser la mélatonine, les producteurs $\mathrm{AB}$ sont obligés d'appliquer des JC artificiels et d'en supporter les inconvénients. Pour les éleveurs qui pratiquent une seule mise bas par an à contre-saison, les traitements lumineux peuvent être appliqués chaque année sans contrainte particulière sur les résultats de reproduction ultérieurs. Toutefois, dans le cas où les éleveurs souhaitent 2 périodes de mises bas ou plus, il est nécessaire de disposer de plusieurs bâtiments afin d'éviter des interactions entre les traitements des différents lots. Les races peu saisonnées permettraient de s'affranchir de traitements lumineux pour les mises à la reproduction à contre-saison et seraient les mieux adaptées si l'objectif est de réussir 2 périodes ou plus de mises bas par an. Dans le cas particulier des chèvres laitières françaises (très saisonnées), la pratique de mises bas tardives peut également s'avérer une bonne solution pour optimiser la production de lait d'automne sans recours aux hormones ni aux traitements lumineux.

Les éleveurs $\mathrm{AB}$ peuvent donc bénéficier des schémas de sélection car l'IA est autorisée. Toutefois, la mise en place de l'IA doit se faire sans synchronisation hormonale des ovulations. Cela implique la pratique systématique de la détection de chaleurs sur plusieurs jours pour identifier les femelles à inséminer en l'absence de groupage de chaleurs. Cette pratique peut être appliquée soit pendant la saison sexuelle, soit à contre-saison sur ovulations stimulées par un traitement lumineux. Les éleveurs peuvent aussi utiliser l'effet mâle pour synchroniser les chaleurs et ainsi réduire le nombre de moments d'IA par lot, mais seulement en dehors de la saison sexuelle.

En ovin allaitant $A B$, l'effet mâle est une pratique assez répandue. $\mathrm{Au}$ contraire, les traitements lumineux sont d'un usage anecdotique. Enfin, si le flushing est fréquemment utilisé pour accroître la prolificité, l'accélération du rythme de mises bas pour améliorer la productivité numérique est une conduite assez peu répandue car, dans les conditions de l'AB, son intérêt économique est limité (Benoit et al 2009).

En ovin laitier AB, l'effet mâle est vraisemblablement mis en pratique pour les luttes de contre-saison (principalement en mai dans les Pyrénées Atlantiques mais aussi en Aveyron). Des luttes en septembre sont aussi répertoriées (voire pour certains élevages en décembre et jusqu'en février). Les traitements lumineux ne sont pas utilisés en brebis laitières ce qui rend très difficile la lutte en mars et en avril.

En ovin viande et en ovin laitier $A B$, l'IA n'est pas du tout pratiquée, or elle est indispensable pour participer aux schémas de sélection et gérer la consanguinité sans introduction de mâles provenant d'autres élevages.

En élevage caprin $\mathrm{AB}$, la reproduction est surtout assurée par monte naturelle pour obtenir des mises bas de sai- 
Figure 5. Effet des différents facteurs d'élevage sur la réponse à l'effet mâle (Tournadre et al 2002, 2008) :

(Exp. 1) : Influence de la date d'introduction des béliers. D1 : 13 avril, D2 : 28 avril, D3 : 12 mai, D4 : 26 mai.

(Exp. 2) : Influence de l'intervalle tarissement-lutte. $11: 22$ j, $12: 86$ j.

(Exp. 3) : Influence du flushing ( $F$ : traitées, $T$ : témoins) et de l'état corporel avant la lutte (M : NEC 3.0 maigres, G : NEC 3.9 grasses).

a,b,c : exposants différents désignent différences significatives au seuil de $5 \%$.

Exp.1

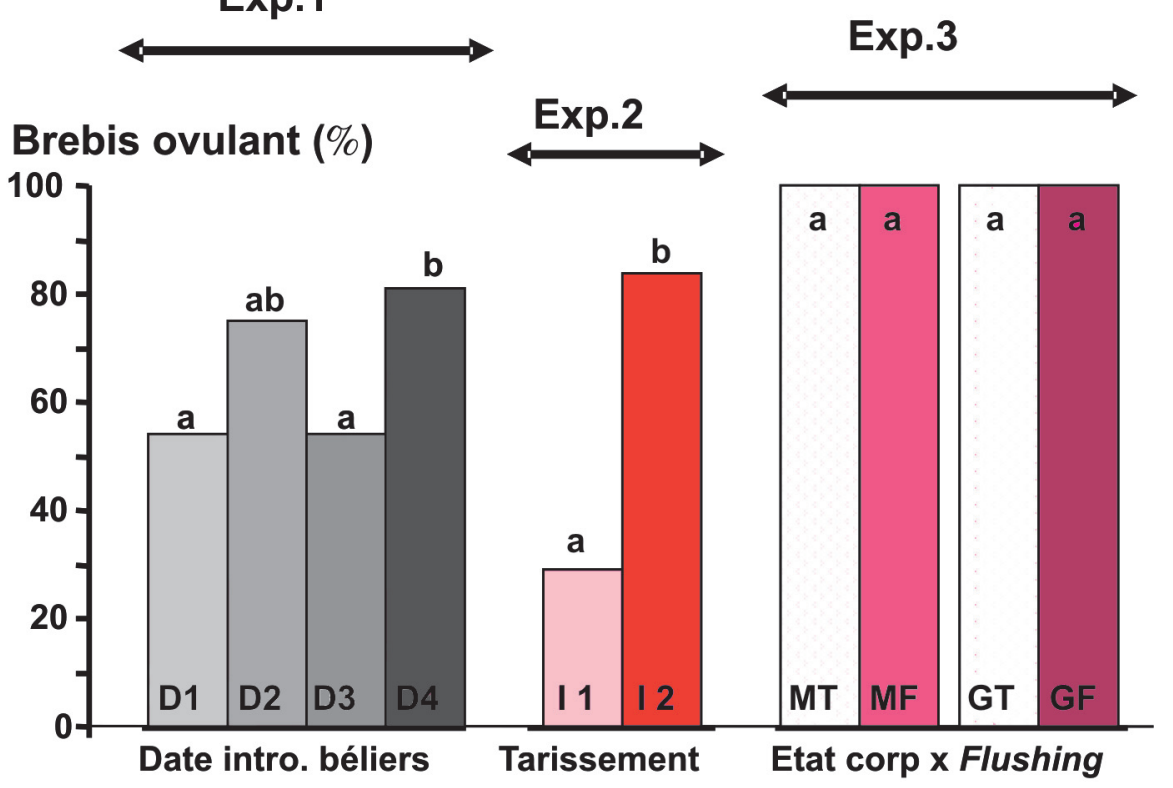

\section{Brebis mettant bas (\%)}

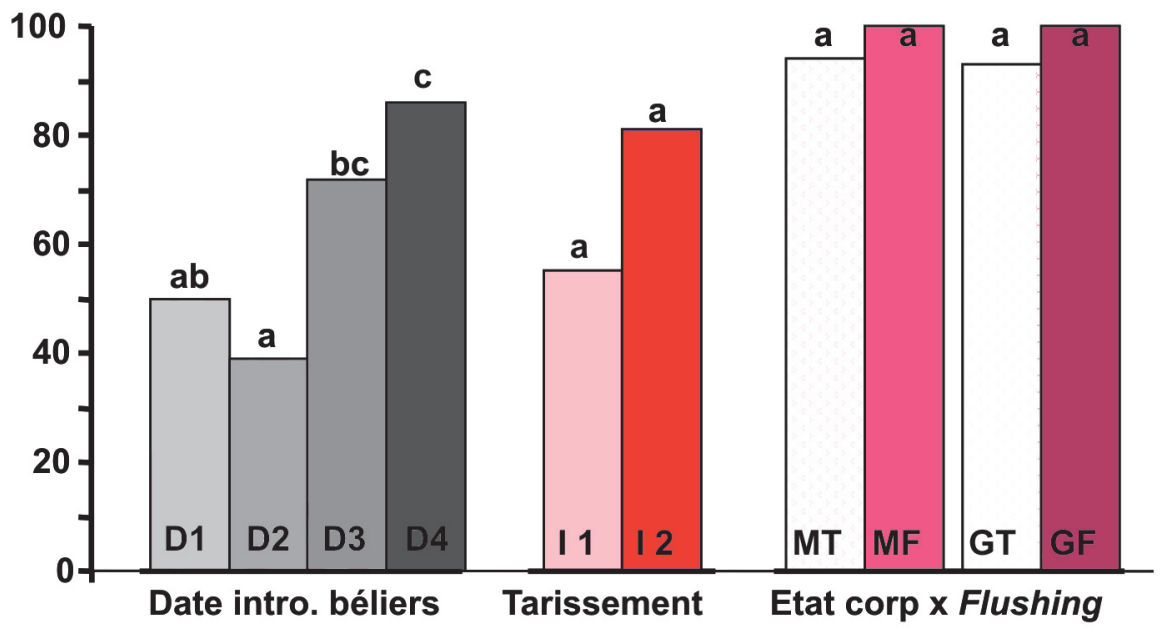

son de janvier à mars, impliquant parfois la mise en pratique d'un effet mâle en fin d'anoestrus saisonnier. Certains éleveurs utilisent les traitements lumineux pour des mises à la reproduction à contre-saison. D'autres mettent aussi en place l'IA sur chaleurs naturelles.

\section{2 / Maîtrise de la reproduc- tion chez les porcins}

La reproduction chez le porc domestique est non saisonnée. La femelle, en agriculture conventionnelle visent à maximiser la taille de la portée, ainsi que le nombre de portées par an. Pour cela, ils font appel à des races hyperprolifiques et réduisent le cycle de production des truies en jouant sur la durée d'allaitement et l'Intervalle SevrageSaillie Fécondante (ISSF). La conduite de la reproduction en bandes est caractéristique de l'élevage de porc. Elle a pour objectifs d'améliorer l'organisation du travail, la surveillance des naissances et de produire des lots de porcelets homogènes au sevrage.

Les lignées les plus utilisées en élevage conventionnel sont les croisées Large White $x$ Landrace. Les truies issues de lignées hyper-prolifiques (Large White) ou sino-européennes (Large White $x$ Landrace $x$ Meishan) permettent d'atteindre des prolificités élevées : 13,9 nés totaux en moyenne par portée (Porc performances 2007). La conduite de ces animaux requiert cependant une grande technicité, en particulier pour compenser l'effet négatif d'une forte prolificité sur la viabilité néonatale et pour gérer les porcelets surnuméraires (Boulot et al 2008).

La conduite majoritaire de l'élevage de porc est dite en 7 bandes espacées de trois semaines, ce qui correspond à la durée d'un cycle ovarien. La durée du cycle d'une bande est de 21 semaines (16 semaines de gestation, 4 semaines d'allaitement et 1 semaine d'ISSF). Il existe aussi, pour les élevages de grande taille ( $>450$ truies) une conduite dite à la semaine (20 ou 21 bandes). Plus rarement, des systèmes de conduite en 3, 4 et 5 bandes sont mis en place afin d'adapter la charge de travail aux disponibilités en main-d'œuvre.

Quelle que soit la conduite en bandes pratiquée, la durée d'allaitement est en général de 4 semaines. Toutefois, le sevrage à 4 semaines tend à régresser au profit du sevrage à 3 semaines pratiqué aujourd'hui par plus de $35 \%$ des élevages (IFIP - GTTT 2008). Il est motivé à la fois par la recherche de gains de productivité (augmentation du rythme de reproduction) et par le souci de limiter les pertes d'état corporel chez les truies allaitant des portées de plus en plus nombreuses.

Le sevrage est utilisé de façon systématique pour la synchronisation des chaleurs des truies en bandes. Néanmoins, des traitements progestatifs à base d'altrenogest (agoniste de synthèse de la progestérone ; Régumate $\AA$, Altresyn () ) sont adminis- 
trés par plus de $80 \%$ des éleveurs pour recycler des truies décalées ou pour intégrer les cochettes de renouvellement dans les bandes. La fréquence de ces interventions dépend du type de conduite (Boulot et al 2005) : elle est réduite pour les conduites en 20 ou 21 bandes qui offrent une grande souplesse de gestion. L'altrenogest est aussi utilisé ponctuellement en lactation pour limiter les venues en chaleurs en maternité. Enfin, l'induction hormonale des mises bas (utilisée dans $80 \%$ des élevages), la stimulation hormonale (gonadotrophines) ou nutritionnelle (flushing) des truies sevrées sont des traitements qui peuvent favoriser le groupage des inséminations et de bons résultats de reproduction.

La conduite de la reproduction en élevage porcin est également basée sur l'application généralisée de l'insémination artificielle. Elle concerne plus de $90 \%$ des truies et permet une fertilité proche de $90 \%$. Elle est pratiquée à partir de doses achetées $(80 \%)$ ou produites sur l'exploitation (Boulot et Badouard 2008). Les truies sont inséminées sur oestrus naturels synchronisés par le sevrage. Les cochettes et les truies décalées sont inséminées sur oestrus synchronisés par traitement hormonal. L'organisation du chantier d'insémination dépend donc de la maîtrise de la conduite en bandes.

\section{2 / En élevage porcin biolo- gique}

Les points principaux de la réglementation sur la production de porc $\mathrm{AB}$ qui affectent les performances de reproduction concernent le principe d'un élevage lié au sol, l'allongement du temps de la lactation et l'interdiction de l'utilisation d'hormones ou de substances analogues pour la maîtrise de la reproduction.

Le mode de production $\mathrm{AB}$ est basé sur le principe d'un élevage lié au sol en interdisant explicitement l'élevage horssol et en imposant l'accès au plein air. Ceci implique des conduites spécifiques en plein air intégral au pâturage ou en logement fermé avec accès à des espaces en plein air (Bonde et Sorensen 2006). La conduite en plein air, constitue un facteur de risque de dégradation des performances de reproduction (Porc performances 2007, tableau 2). Les facteurs de risque liés à l'élevage plein air sont bien connus et seront a priori identiques dans les élevages $\mathrm{AB}$ et conventionnels : des difficultés pour la détection des chaleurs, des avortements et des boiteries liés aux bagarres, une infertilité en relation avec un mauvais état corporel des truies, une infertilité d'été des verrats, une compétition alimentaire, une mauvaise surveillance sanitaire et une mortalité périnatale élevée liée à la difficulté de surveillance des maternités plein air. Les mises bas en plein air ou en cases de maternité libre compliquent aussi la gestion d'une prolificité élevée. Au-delà de 12 porcelets nés vivants, les éleveurs observent une très forte mortalité néonatale (Chambre d'Agriculture de la Mayenne 2003).

Le règlement européen prévoit l'augmentation du temps d'allaitement des porcelets à $40 \mathrm{j}$ minimum. En pratique, du fait de la conduite en bandes et de l'allongement de la lactation, le sevrage a lieu à $42 \mathrm{j}$ et le cycle de reproduction s'étend à 23 semaines. L'obligation d'allonger la durée d'allaitement constitue un facteur susceptible d'accentuer les effets négatifs d'une sous-alimentation protéique sur les truies allaitantes et d'aggraver ainsi la fonte adipeuse et musculaire au cours de la lactation. En effet, les acides aminés de synthèse sont interdits en $A B$ et les protéines d'origine biologique (soja, pois...) ne sont disponibles qu'en quantités limitées. Or, en cas de mobilisation excessive des réserves, les performances de reproduction seront dégradées au cours des cycles ulté-

Tableau 2. Performances des élevages plein air français (IFIP-GTTT, Porc performances 2007).

\begin{tabular}{|l|c|c|}
\hline & Plein air & Bâtiments \\
\hline Nombre d'élevages & 81 & 2742 \\
Sevrés/truie productive/an & 23,8 & 27,0 \\
Nés totaux/portée & 12,2 & 12,8 \\
Sevrés/portée & 10,0 & 11,1 \\
Pertes sur nés totaux \% & 24,1 & 20,6 \\
Age au sevrage (jours) & 27,6 & 24,6 \\
Intervalle sevrage-saillie fécondante (j) & 10,3 & 8,9 \\
Taux de fécondation en saillie 1'ire $\%$ & 86,4 & 89,0 \\
\hline
\end{tabular}

rieurs (anoestrus, infertilité, petites portées). En outre, une lactation longue ( $>5$ semaines) ne semble pas adaptée aux tailles de portée élevées. Du fait de l'allaitement de $42 \mathrm{j}$, il est conseillé d'éviter les truies de lignées hyper-prolifiques utilisées en élevages conventionnels. L'objectif d'un éleveur de porc $\mathrm{AB}$ est donc de sevrer 9 à 10 porcelets pour limiter la fonte adipeuse et musculaire durant l'allaitement.

L'efficacité de la conduite en bandes est pénalisée en $\mathrm{AB}$ par l'interdiction des traitements hormonaux utilisés fréquemment en conventionnel pour intégrer les cochettes dans les bandes, pour recycler des femelles décalées ou pour prévenir des oestrus de lactation. Aussi, sans recours aux hormones gonadotropes en cas d'anoestrus (Boulot et al 2005), les temps improductifs peuvent se voir allongés de façon importante. Cependant, l'interdiction de l'utilisation d'hormones ne pénalise pas la pratique de l'IA car elle est réalisée sur oestrus naturels synchronisés par le sevrage chez la majorité des producteurs en $\mathrm{AB}$ et en élevage conventionnel. L'insémination artificielle est autorisée en $\mathrm{AB}$ sans restriction sur l'origine des mâles (achat en CIA ou doses fabriquées par les éleveurs). A défaut de mention spécifique, a priori, tous les types de doses (diluées, congelées), d'additifs autorisés pour les CIA (conservateurs, antibiotiques...) et de matériels de mise en place sont utilisables.

L'ensemble de ces difficultés auxquelles doit faire face l'éleveur de porcs en $\mathrm{AB}$ se traduit par une diminution des performances de reproduction en $\mathrm{AB}$ par rapport aux troupeaux conventionnels (Lauritsen et al 2000, Hermansen 2005, tableau 3). Certaines pratiques d'élevage concernant la gestion de la prolificité, le déclenchement et la synchronisation de la puberté et la prévention des oestrus de lactation, peuvent toutefois êtres utilisées pour pallier cette diminution de productivité.

Pour limiter la prolificité, bien qu'il n'y ait pas d'obligation dans le choix des races en élevage $A B$, certains éleveurs se sont orientés vers l'utilisation de races locales moins prolifiques et produisant des carcasses de moindre qualité, mais plus adaptées au milieu. La création de lignées adaptées aux élevages alternatifs (sélection sur de nouveaux critères, gènes issus des races locales) pourrait améliorer les performances, mais les objectifs de sélection restent à préciser. 
Les éleveurs en $\mathrm{AB}$ sont confrontés à de grandes difficultés pour intégrer les cochettes dans les bandes. Les cochettes sont donc saillies ou inséminées sur chaleurs naturelles puis conduites hors bande pendant leur première lactation. Elles n'intègrent les lots qu'au moment du sevrage. Ceci peut induire des allaitements encore plus longs préjudiciables à la carrière de ces jeunes reproductrices. L'effet verrat et le stress du transport sont des méthodes alternatives qui pourraient favoriser le déclenchement et la synchronisation de la puberté. L'exposition au verrat permet de stimuler et de mieux grouper l'apparition de la puberté (Hughes et Thorogood 1999). Toutefois, «l'effet verrat» est une pratique très peu utilisée en élevage. Le transport a pour effet l'induction et la synchronisation de l'oestrus des cochettes pré-pubères (Hughes 1982). En auto-renouvellement, un transport «artificiel» est parfois effectué pour induire le premier oestrus.

Bien que les éleveurs en AB utilisent le sevrage pour synchroniser les venues en chaleurs des truies dans leur bande, comme dans le cas d'un élevage conventionnel, la venue en oestrus de certaines femelles peut avoir lieu en maternité durant la lactation du fait du prolongement de la lactation au delà de $40 \mathrm{j}$. Ceci peut concerner jusqu'à $33 \%$ des truies au delà de $35 \mathrm{j}$ de lactation (Hulten et al 2006). Ces femelles se retrouvent alors décyclées par rapport à leur bande. La prévention non hormonale des chaleurs de lactation est ainsi un objectif des éleveurs de porc en $\mathrm{AB}$ pour améliorer la maîtrise de la conduite en bandes. En élevage conventionnel, cet objectif repose largement sur le choix de truies peu sensibles au stress, la limitation du stress des truies allaitantes, le maintien d'une production laitière élevée avec des porcelets actifs à la mamelle et peu de mortalité (Hulten et al 2006). Toutefois, il apparaît que ces recommandations ne sont pas suffisantes à cause des lactations excessivement longues dans les éleva- ges AB. Les traitements photopériodiques pourraient aussi être utilisés pour maîtriser les venues en chaleurs en lactation ou après sevrage. Par exemple, l'intervalle sevrage-oestrus peut être rallongé si le sevrage a lieu pendant un traitement de jours longs (Prunier et al 1994, Hälli et al 2008).

\section{3 / Maîtrise de la reproduc- tion chez les bovins}

La carrière reproductrice de la vache commence à la puberté, qui intervient chez les génisses laitières entre 10 et 15 mois d'âge (lorsque la génisse a atteint 40 à $50 \%$ du poids adulte) et plus tardivement chez les génisses allaitantes, après 15 mois (lorsque la génisse a atteint 55 à $60 \%$ du poids adulte). Après la puberté, les cycles de reproduction sont caractérisés par la succession des phases suivantes : activité ovarienne cyclique (les cycles oestraux durent en moyenne $21 \mathrm{j}$ ), mise à la reproduction, gestation, vêlage, anoestrus post-partum puis retour à l'activité ovarienne cyclique. La gestation dure en moyenne 9 mois et une semaine, avec des variations selon les races (par exemple les durées de gestation de référence par race sont de $282 \mathrm{j}$ en Prim'Holstein et $287 \mathrm{j}$ en Charolaise). Après la mise bas, la période du postpartum est caractérisée par l'involution utérine, le démarrage de la lactation et la reprise de l'activité ovarienne cyclique après une phase plus ou moins longue d'anoestrus, plus marqué chez les vaches allaitantes (Humblot et Grimard 1996, Grimard et Disenhaus 2005). Le terme d'anoestrus post-partum recouvre à la fois les phénomènes d'inactivité ovarienne (femelle non cyclée) et de chaleurs non observées alors que l'ovulation a eu lieu (ovulation silencieuse ou chaleurs non détectées). Physiologiquement, les bovins ne sont pas des animaux dont la reproduction est saisonnée, mais ils sont aussi sensibles à la photopériode. Un effet de la saison de mise à la reproduction peut

Tableau 3. Performances des élevages biologiques Danois (Lauritsen et al 2000).

\begin{tabular}{|l|c|c|}
\hline & $\begin{array}{c}\text { Elevages } \\
\text { biologiques } \\
(n=4,1998)\end{array}$ & $\begin{array}{c}\text { Elevages } \\
\text { conventionnels } \\
\text { (Danemark, 1998) }\end{array}$ \\
\hline Sevrés/truie/an & 17,0 & 23,0 \\
Nés totaux/portée & 11,0 & 11,5 \\
Sevrés/portée & 9,4 & 10,2 \\
Taux de pertes/nés vivants \% & 14,7 & 12,0 \\
Taux de mise bas \% & 74 & 85 \\
\hline
\end{tabular}

être observé sur la fertilité. Cet effet est multifactoriel : production laitière ou allaitante, type de logement, ressources alimentaires, température, photopériodisme, etc. La mise à la reproduction est possible en toutes saisons avec des atouts et des contraintes différents selon les objectifs de production (Disenhaus et al 2005). Toutefois, par modélisation, il a été mis en évidence que la date de vêlage influence l'intervalle vêlage-saillie fécondante, ce qui peut être en partie expliqué par la photopériode (Blanc et Agabriel 2008). En outre, la manipulation de la photopériode pendant la lactation et la période de tarissement semble intéressante pour optimiser la production laitière dans certains contextes d'élevage ; la photopériode exerce également une influence sur le développement corporel et mammaire, sur la puberté chez les génisses (Dahl et al 2000, Serieys 2007) et sur la durée du post-partum chez les vaches allaitantes (Garel et al 1987). Enfin, de façon similaire à l'effet mâle observé chez les petits ruminants, en troupeaux allaitants, l'exposition au taureau peut permettre d'augmenter la proportion de génisses pubères au moment de la mise à la reproduction (Roberson et al 1991). Cette exposition peut aussi permettre de réduire la durée de l'anoestrus post-partum (Monje et al 1992, Cupp et al 1993).

\section{1 / En élevage bovin conven- tionnel}

Chez les bovins, l'insémination naturelle ou artificielle est pratiquée de manière générale sur chaleurs naturelles :

- monte naturelle : un taureau est mis en présence d'un groupe de femelles à saillir (c'est le taureau qui détecte les femelles en chaleur) ;

- insémination artificielle : c'est l'éleveur qui détecte les chaleurs des femelles par l'observation de signes comportementaux et physiques, en ayant éventuellement recours à des outils d'aide à la détection (Saint-Dizier 2005, Disenhaus 2008) et appelle l'inséminateur qui va mettre en place la semence congelée (Gérard et al 2008).

En troupeaux laitiers, le taux d'utilisation de l'IA (nombre d'inséminations premières (IAP)/cheptel total) est d'environ $85 \%$ alors qu'il n'est que d'environ $17 \%$ en troupeaux allaitants (France Génétique Elevage 2007). Une bonne détection des chaleurs par l'éleveur ainsi qu'une contention des femelles sont nécessaires à la pratique de l'IA : ces contraintes expliquent en 
grande partie son faible développement dans les troupeaux allaitants. La mise à la reproduction des vaches après la mise à l'herbe est un frein au développement de l'IA.

Chez la vache comme chez la génisse, l'utilisation de traitements hormonaux permet d'induire et de synchroniser l'ovulation et le moment d'apparition des chaleurs. L'intérêt est de pouvoir pratiquer l'IA sans détection préalable des chaleurs sur un lot de femelles, de choisir la période de mise à la reproduction des génisses et donc leur période de vêlage (qui conditionne celle du troupeau entier), de réduire l'intervalle vêlage-IA fécondante sur des femelles en anoestrus (réduction de l'intervalle entre vêlages successifs, avancement de la période de vêlage suivante...), de pratiquer une conduite en bandes, de synchroniser les chaleurs des receveuses dans le cadre de la transplantation embryonnaire, etc.

Les traitements disponibles actuellement en France sont à base de prostaglandine F2 $\alpha$ (ou analogue) ou de progestagènes. Leurs modes d'action et les résultats de fertilité après oestrus induit ne sont pas détaillés ici (Grimard et al 2003, Picard-Hagen et al 2005, Ponsart et al 2005) :

- le protocole PGF2 $\alpha$ (double injection de PGF2 $\alpha$ à 11-14 j d'intervalle), et le protocole GnRH/PGF $2 \alpha / \mathrm{GnRH}$ (3 injections) sont utilisables chez les femelles cyclées uniquement.

- les dispositifs à base de progestérone naturelle (spirale vaginale PRID $\mathbb{R}$ ou dispositif intravaginal CIDR $\AA$ ) ou de norgestomet (implant auriculaire CRESTAR $(S O)$ sont associés à une injection systématique de PGF2 $\alpha 24$ à $48 \mathrm{~h}$ avant retrait (selon la spécialité), à une injection d'eCG au retrait chez les femelles non cyclées (pour PRID ${ }^{\circledR}$ et CRESTAR ${ }^{R} S O$ ), et à une injection de buséréline (analogue de $\mathrm{GnRH}$ ) à la pose pour CRESTAR $R S O$. Ils sont utilisables chez les femelles cyclées et

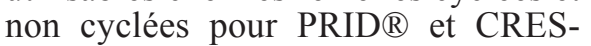
TAR ${ }^{R S O}$ (avec IA à heure fixe après retrait) et uniquement chez les femelles cyclées pour CIDR $\AA$ (avec IA sur chaleurs observées après retrait).

Le nombre total de traitements pro-

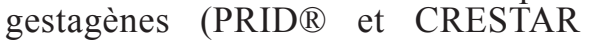
(RSO) utilisés en France en 2007 sur les vaches laitières et allaitantes était de l'ordre de 450000 (Picard-Hagen et al 2009, source CEVA et Intervet). Pour avoir une estimation de la fréquence d'utilisation de ces traitements, il est possible de rapporter ce nombre au cheptel total de vaches laitières et allaitantes en 2007 (environ 7876 000, France Génétique Elevage 2007), ce qui donne 5,7\%.

Sur la zone de la coopérative d'insémination COOPELSO (région SudOuest), qui correspond à une population de plus de 100000 vaches laitières et 80000 vaches allaitantes inséminées, le bilan du programme de synchronisation de la reproduction sur l'exercice 2006-2007 montre que l'utilisation des traitements de maîtrise des cycles reste marginale en élevage laitier $(1,9 \%)$ et est plus développée en élevage allaitant (9,3\%) (Picard-Hagen et al 2009).

\section{2 / En élevage bovin biologique}

La mise à la reproduction étant essentiellement assurée par insémination artificielle sur chaleurs naturelles ou par monte naturelle en élevage bovin conventionnel ou biologique, l'interdiction d'utilisation des traitements hormonaux d'induction et de synchronisation des chaleurs ne pose pas de réel problème en élevage biologique. D'un point de vue zootechnique, il n'y a pas de différence majeure de conduite de la reproduction en élevage biologique par rapport à l'élevage conventionnel.

La comparaison des résultats technico-économiques 2004 entre 22 exploitations allaitantes certifiées AB (suivies dans le cadre du réseau de fermes de références bovin allaitant en $\mathrm{AB}$ dans le Massif Central) et 400 exploitations conventionnelles du socle national des réseaux d'élevage montre que les performances de reproduction (taux de gestation et productivité numérique) ne sont pas pénalisées en élevage biologique (Veysset et al 2006).

Depuis 1994, le dispositif des Réseaux d'Elevage pour le Conseil et la Prospective intègre des suivis d'exploitations biologiques dans la plupart des grandes régions d'élevages, permettant de disposer de plusieurs années de suivis sur un grand nombre d'exploitations bovin lait et bovin allaitant certifiées et commercialisant en circuit biologique (Pavie et Retif 2006). Ainsi, des données sur les performances de reproduction ont pu être analysées sur la période 2000-2006 dans deux échantillons constants : 20 exploitations bovin lait (races principales : Prim'Holstein, Normande, Montbéliarde) et 20 exploitations bovin viande (races principales : Limousine, Charolaise, Blonde d'Aquitaine) en AB
(Pavie, données non publiées). En 2006, dans l'échantillon bovin allaitant, l'utilisation de l'insémination artificielle comme unique mode de reproduction a augmenté mais ne concerne que $10 \%$ des exploitations étudiées, $60 \%$ des éleveurs ont uniquement recours aux saillies naturelles. Les $30 \%$ restant pratiquent les deux méthodes. Malgré sa progression, l'insémination artificielle seule reste peu pratiquée dans les élevages allaitants, et ce, aussi bien en élevage biologique qu'en conventionnel. Dans l'échantillon bovin lait, 50\% des éleveurs biologiques utilisent exclusivement l'insémination artificielle. La part d'éleveurs dont le troupeau n'est mené qu'en saillie naturelle est faible (de l'ordre de 10\%). De nombreux troupeaux restent conduits de manière mixte : insémination artificielle et taureau (un taureau dit «de rattrapage» est fréquemment utilisé pour les retours).

Les performances de reproduction des 2 échantillons AB pour les années 2004 à 2006, ainsi que les résultats moyens observés en élevages conventionnels pour une partie des critères, sont présentés dans le tableau 4 : les résultats de reproduction des troupeaux biologiques sont assez proches de ceux des conventionnels. En bovin allaitant, la comparaison des répartitions mensuelles des vêlages montre que les pratiques biologiques et conventionnelles sont comparables. En bovin lait, cette comparaison montre des différences de pratiques. La place prépondérante de l'herbe dans les systèmes biologiques oriente des stratégies d'exploitations vers le vêlage de printemps (mais c'est la période où le prix est le plus bas du marché). Les éleveurs conventionnels, dont l'alimentation hivernale du troupeau n'est pas forcément un facteur limitant (recours au maïs notamment) privilégient les vêlages d'automnehiver pour bénéficier du point haut des cours du lait (Pavie, données non publiées).

\section{4 / Perspectives}

Malgré la disponibilité de méthodes efficaces et utilisables en AB pour rendre possible la reproduction à contresaison (traitements lumineux, effet mâle), la maîtrise de la saisonnalité reste l'une des principales préoccupations dans les élevages de petits ruminants en $\mathrm{AB}$. Concernant le recours au photopériodisme, nous avons évoqué les difficultés et contraintes associées à l'application de jours courts artificiels en bâtiment ouvert pour des mises à la 
reproduction de juin à septembre. Ceci constitue un frein important à la généralisation de cette pratique. La mise au point de nouveaux traitements lumineux alternatifs aux JC artificiels et sans mélatonine faciliterait l'appropriation de cette technique par les éleveurs ovins et caprins en AB. Par ailleurs, pour mimer les jours courts il serait aussi possible de faire appel à la phytothérapie grâce à des plantes contenant de la mélatonine (Dubbels et al 1995). L'état nutritionnel des animaux joue aussi un rôle sur la durée de la saison sexuelle (Scaramuzzi et Martin 2008), des stratégies nutritionnelles peuvent ainsi être envisagées pour allonger la période de reproduction. De plus, la sélection génétique est envisageable pour réduire la saisonnalité de nos races car les dates de début et de fin de la saison sexuelle sont des caractères héritables (Chemineau et al 2008).
La variabilité de la réponse à l'effet mâle que l'on observe entre élevages et entre années est le principal frein à l'utilisation de cette technique chez les petits ruminants, notamment en cas d'IA. La généralisation de la pratique de l'effet mâle pourrait se voir facilitée par une meilleure connaissance des facteurs physiologiques et environnementaux qui agissent sur la qualité de la réponse à l'effet mâle (Scaramuzzi et Martin 2008, Tournadre et al 2008). La maîtrise de cycles courts induits par effet mâle est l'un des objectifs prioritaires pour l'IA. En effet, la possibilité d'induire chez toutes les femelles soit un premier cycle court, soit un premier cycle normal, permettrait d'obtenir un seul pic d'ovulations fertiles et de réduire ainsi au minimum le nombre d'IA nécessaires pour maximiser les résultats de fertilité. Parmi les facteurs environnementaux autres que la photo- période, la nutrition prend à nouveau une place privilégiée comme moyen de moduler l'efficacité de l'effet mâle chez les petits ruminants (Scaramuzzi et Martin 2008).

Des études de conservation de la semence sont aussi nécessaires chez les petits ruminants pour améliorer l'aptitude à la fécondation des spermatozoïdes sur des durées supérieures à $24 \mathrm{~h}$ dans le tractus femelle. Cette approche est très intéressante comme technique d'appui pour le développement de l'IA sans hormone sur des lots de femelles dont le degré de synchronisation des ovulations est plus faible que pour celles traitées avec des hormones.

Chez les porcins, les recherches en $\mathrm{AB}$ devraient s'orienter principalement vers l'optimisation de la conduite en bandes sans hormones. Par exemple, la

Tableau 4. Performances de reproduction d'élevages bovins laitiers et allaitants biologiques (Pavie données non publiées).

\begin{tabular}{|c|c|c|c|c|}
\hline \multicolumn{5}{|c|}{ Bilan de reproduction échantillon constant bovins viande $\mathrm{AB}$ ( $n=20$ exploitations) } \\
\hline & 2004 & 2005 & 2006 & $\begin{array}{l}\text { données moyennes } \\
\text { en élevages conventionnels }\end{array}$ \\
\hline Taux de renouvellement $(\%)$ & 23 & 22 & 22 & \\
\hline $\begin{array}{l}\text { Age moyen au } 1^{e r} \text { vêlage } \\
\text { (mois) }\end{array}$ & 36 & 36 & 36 & $\begin{array}{l}33 \text { à } 35 \text { mois (France Bovins } \\
\text { Croissance 2007) }\end{array}$ \\
\hline IVV moyen (j) & 381 & 387 & 376 & \\
\hline$\%$ d'IVV > $400 \mathrm{j}$ & 20 & 25 & 17 & $\begin{array}{l}<25 \% \text { en Charolaise et Limousine, } \\
36 \% \text { en Blonde d'Aquitaine } \\
\text { (France Bovins Croissance 2007) }\end{array}$ \\
\hline Taux de gestation (\%) & 90 & 93 & 92 & $95 \%$ (Veysset et al 2006) \\
\hline Taux de prolificité (\%) & 102 & 103 & 104 & \\
\hline Taux de mortalité (\%) & 11 & 6 & 8 & \\
\hline $\begin{array}{l}\text { Taux de productivité } \\
\text { numérique }(\%)\end{array}$ & 81 & 89 & 88 & $88 \%$ (Veysset et al 2006) \\
\hline \multicolumn{5}{|c|}{ Bilan de reproduction échantillon constant bovins lait $\mathrm{AB}$ ( $n=20$ exploitations) } \\
\hline & 2004 & 2005 & 2006 & $\begin{array}{l}\text { données moyennes } \\
\text { en élevages conventionnels }\end{array}$ \\
\hline Rang moyen de lactation & 2.9 & 2.9 & 2.9 & 2.5 (France Contrôle Laitier 2007) \\
\hline $\begin{array}{l}\text { Age moyen au } 1^{\text {er }} \text { vêlage } \\
\text { (mois) }\end{array}$ & 35 & 35 & 34 & $\begin{array}{l}30 \text { mois en Prim'Holstein et } 33 \text { mois } \\
\text { en Normande et Montbéliarde } \\
\text { (France Contrôle Laitier 2007) }\end{array}$ \\
\hline$\%$ de réussite en $1^{\text {ère }} \mid \mathrm{A}$ & 62 & 50 & 48 & $\begin{array}{l}50 \text { à } 55 \% \text { en Montbéliarde, environ } \\
50 \% \text { en Normande et } 35 \text { à } 40 \% \\
\text { en Prim'Holstein (Le Mezec et Barbat 2007) }\end{array}$ \\
\hline$\%$ d'intervalle $\mathrm{V}-\mathrm{I} 1$ > $90 \mathrm{j}$ & 27 & 25 & 24 & $\begin{array}{l}75 \text { j en Montbéliarde, } 77 \text { j en Normande, } \\
88 \text { j en Prim'Holstein } \\
\text { (Le Mezec et Barbat 2007) }\end{array}$ \\
\hline$\%$ d'intervalle V-IF > $110 \mathrm{j}$ & 39 & 37 & 39 & $\begin{array}{l}101 \text { j en Montbéliarde, } 103 \text { j en Normande, } \\
128 \text { j en Prim'Holstein } \\
\text { (Le Mezec et Barbat 2007) }\end{array}$ \\
\hline IVV moyen (j) & 395 & 391 & 385 & $\begin{array}{l}386 \text { j en Montbéliarde, } 388 \text { j en Normande, } \\
408 \text { j en Prim'Holstein } \\
\text { (Le Mezec et Barbat 2007) }\end{array}$ \\
\hline$\%$ d'IVV > 400 j & 24 & 20 & 25 & \\
\hline Taux de renouvellement $(\%)$ & 26 & 27 & 26 & \\
\hline
\end{tabular}


manipulation de la photopériode semble intéressante pour maîtriser les venues en oestrus en lactation ou après sevrage. Pour cela, il sera nécessaire de mieux préciser l'effet de la photopériode sur la reprise de l'activité ovulatoire post-partum et l'expression des chaleurs, pour ensuite pouvoir développer des traitements efficaces applicables en ferme. L'effet verrat, est aussi une alternative intéressante pour favoriser le déclenchement de la puberté des cochettes (Hughes et Thorogood 1999), améliorer l'expression des chaleurs voire agir sur le moment d'ovulation chez la truie (Kemp et al 2005 ) et réduire l'infertilité saisonnière (Paterson et al 1991). Toutefois, les pratiques permettant une synchronisation des chaleurs efficace restent à définir.

Des approches multi-espèces sont aussi envisageables. La qualité de la détection des oestrus est essentielle en élevage porcin pour optimiser la conduite en bandes, encore plus dans des élevages en plein air. Il en est de même chez les petits ruminants pour l'IA sans hormones. Dans les élevages bovins, la détection des chaleurs est aussi une opération clé de la conduite de la reproduction. La détection de l'oestrus repose aujourd'hui sur le savoir faire des éleveurs et l'intensité des signes exprimés. Des approches technologiques telles que les récents systèmes automatiques d'assistance à la détection de l'oestrus sont en voie de développement et sont très prometteurs (Bocquier et al 2006, Maton et al 2008). De plus, chez les bovins, un programme de recherche-développement (financé par le CASDAR) est actuellement en cours sur l'amélioration de la détection des chaleurs dans les troupeaux bovins, laitiers et allaitants, dans le cadre d'un partenariat Institut de l'Elevage, UNCEIA, INRA, ENITA Clermont-Ferrand, AgroCampus Rennes, ENV Nantes et ENV Alfort.
Enfin, l'intérêt d'extraits nutritionnels naturels (additifs ou plantes présentes dans les pâturages) et de la phytothérapie pour la maîtrise de la reproduction est en général peu étudié chez les mammifères d'élevage (Kis et Bilkei 2003). Toutefois, il existe des plantes médicinales capables d'agir et de moduler l'activité de l'axe hypothalamo-hypophysaire et/ou l'activité gonadique (Telefo et al 1998). Le développement de la phytothérapie est une autre voie de recherche, certes très ambitieuse mais qui pourrait aboutir à des stratégies et des applications multiples pour la maîtrise de la reproduction des mammifères d'élevage.

\section{Conclusions}

La maîtrise de la reproduction est une pratique d'élevage qui contribue à optimiser la productivité de l'exploitation. La réglementation du mode de production AB n'impose pas de conduites de reproduction spécifiques, mais limite fortement les pratiques autorisées parmi l'ensemble de stratégies employées par les éleveurs aujourd'hui. Les conséquences de ces limitations sont plus ou moins pénalisantes selon l'espèce animale considérée.

Les voies de recherche pour le développement de méthodes naturelles de maîtrise de la reproduction utilisables en $\mathrm{AB}$ sont très diverses, multidisciplinaires et souvent communes aux différentes espèces de mammifères d'élevage. L'INRA contribue fortement à l'amélioration des méthodes existantes et à leur adaptation aux contraintes d'élevage. Il s'implique également dans la conception et la validation de nouvelles approches de maîtrise de la reproduction sans hormones. Ce développement trouvera sa pleine efficacité, non pas dans l'utilisation de telle ou telle méthode, mais dans la combinaison de techniques et/ou d'approches. Il ne pourra connaître un véritable essor que dans le cadre d'une relation étroite entre la recherche finalisée et les professionnels. Des progrès importants restent à accomplir en recherche fondamentale, c'est pourquoi le domaine de la reproduction animale interagit notamment avec ceux de la génétique et de la nutrition, etc. Dans ce contexte, de nouvelles technologies (automatismes, biocapteurs) et la modélisation des nombreux processus mis en œuvre (Blanc et al 2001, Blanc et Agabriel 2008) offrent des perspectives de recherche prometteuses. Les coopérations doivent être actives et itératives pour gagner en efficacité et aboutir à des applications concrètes et acceptables par les professionnels. De plus, les avancées en maîtrise de la reproduction en $\mathrm{AB}$ seront profitables à l'ensemble des élevages dans l'hypothèse d'une interdiction totale de l'utilisation des hormones exogènes en production animale, afin de contribuer à la gestion durable de l'agriculture (Blanc et al 2006).

\section{Remerciements}

Nicole Bossis et Vincent Bellet (Institut de l'Elevage), Gilles Lagriffoul (Institut de l'Elevage, ANIO), Francis Fidelle (CDEO), Blandine Jouve et Michel Albaret (Ovitest), Mathilde Marey (Confédération de Roquefort), Olivier Patout et Hubert Hiron (AVEM) pour leurs renseignements sur les pratiques de maîtrise de la reproduction mises en oeuvre aujourd'hui dans les élevages de petits ruminants en $\mathrm{AB}$ et en conventionnel.

Patrick Veysset (INRA Theix, Unité Economie de l'Elevage) pour ses informations sur les élevages bovins allaitants biologiques et Loys Bodin (INRA, SAGA, Toulouse).

\section{Références}

Baril G., Chemineau P., Cognié Y., Guérin Y, Leboeuf B., Orgeur P., 1993. Manuel de formation pour l'insémination artificielle chez les ovins et caprins. Etude FAO Production et Santé Animales, 83, Rome, Italie, 231

Benoit M., Tournadre H., Dulphy J.P., Laignel G., Prache S., Cabaret J., 2009. Is intensification of reproduction rhythm sustainable in an organic sheep production system? A four-year interdisciplinary study. Animal, 3, 753-763.

Blanc F., Agabriel J., 2008. Modelling the reproductive efficiency in a beef cow herd: effect of calving date, bull exposure and body condition at calving on the calving-conception interval and calving distribution. J. Agric. Sci., 146, 143161.

Blanc F., Martin G.B., Bocquier F., 2001. Modelling reproduction in farm animals: a review. Reprod. Fertil. Dev., 13, 337-353.

Blanc F., Bocquier F., D'Hourd P., Chilliard Y., 2006. Adaptive abilities of the females and sustainability of ruminant livestock systems. A review. Anim. Res., 55, 489-510.
Bocquier F., Gaubert J.L., Blanc F., Viudes G., Maton C., Debus N., Teyssier J., 2006. Utilisation de l'identification électronique pour la détection automatisée du comportement sexuel chez les ovins : perspectives pour la détection des chaleurs chez la brebis. Renc. Rech. Rum., 13, 155-158.

Bonde M., Sorensen J.T., 2006. Animal health and welfare in organic European pig production: State of the art and challenges for the future, based on a North-western European questionnaire survey . Paper presented at Joint 
Organic Congress, Odense, Denmark, May 3031,3 p.

Bonnes G., Desclaude J., Drogoul C., Gadoud R., Jussiau R., Le Loc'h A., Montméas L., Robin $\mathrm{G}$, 2005. Reproduction des animaux d'élevage. $2^{\text {eme }}$ Edition. Educagri Editions, Dijon, France, 407.

Bossis N., Caramelle-Holtz E., Cremoux R., Guinamard C., 2008. Maîtrise de la saisonnalité, produire du lait au bon moment pour répondre aux attentes des entreprises et des éleveurs. Collection résultats, Institut de l'Elevage, Office de l'Elevage, mai, 44.

Boulot S., Badouard B., 2008. Evolution des pratiques d'insémination dans les troupeaux de truies entre 2001 et 2006. Journ. Rech. Porcine Fr., 40, 257-258.

Boulot S., Dubroca S., Badouard B., 2005. Gestion pharmacologique de la reproduction : le point sur les pratiques des éleveurs. Techniporc, 28.

Boulot S., Quesnel H., Quiniou N., 2008. Management of high prolificacy in French herds: can we alleviate side effects on piglet survival? Proc. 37th Banff porc Seminar, Advances in Pork Production, 19, 213-220.

Brice G., 2003 Le désaisonnement lumineux en production caprine. Institut de l'Elevage, 40.

Chambre d'Agriculture de la Mayenne et ITP, 1993. L'élevage plein air, une technique toujours d'actualité. Rapport de synthèse.

Chemineau P., 1989. L'effet bouc : mode d'action et efficacité pour stimuler la reproduction des chèvres en anoestrus. INRA Prod. Anim., 2, 97-104.

Chemineau P., Malpaux B., 1998. Mélatonine et reproduction chez les mammifères d'élevage. C. R. Soc. Biol., 192, 669-682.

Chemineau P., Normant E., Ravault J.P., Thimonier J., 1986. Induction and persistence of pituitary and ovarian activity in the out-of-season lactating dairy goats after a treatment combining a skeleton photoperiod, melatonin and the male effect. J. Reprod. Fertil., 78, 497-504.

Chemineau P., Malpaux B., Guérin Y., Maurice F., Daveau A., Pelletier J., 1992. Lumière et mélatonine pour la maîtrise de la reproduction des ovins et des caprins. Ann. Zootech., 41, 247-261.

Chemineau P., Cognie Y., Heyman Y., 1996a. Maîtrise de la reproduction des mammifères domestiques. 50 ans de recherches en productions animales. INRA Prod. Anim., Hors-Série, 5-15.

Chemineau P., Malpaux B., Pelletier J., Leboeuf B., Delgadillo J.A., Deletang F., Pobel T., Brice G., 1996b. Emploi des implants de mélatonine et des traitements photopériodiques pour maîtriser la reproduction saisonnière chez les ovins et caprins. INRA Prod. Anim., 9, 45-60.

Chemineau P., Baril G., Leboeuf B., Maurel M.C., Roy F., Pellicer-Rubio M., Malpaux B., Cognie Y., 1999. Implications des progrès récents en physiologie de la reproduction pour la conduite de la reproduction dans l'espèce caprine. INRA Prod. Anim., 12, 135-146.

Chemineau P., Guillaume D., Migaud M., Thiéry J.C., Pellicer-Rubio M.T., Malpaux B., 2008. Seasonality of reproduction in mammals: intimate regulatory mechanisms and practical implications. Reprod. Dom. Anim., 43 (suppl.2), $1-8$.

Corke D.G., 1982. Use of teasing in an A.I. programme. Proc. Austr. Soc. Anim. Prod., 13, 81-82.
Cupp A.S., Roberson M.S., Stumpf T.T., Wolfe M.W., Werth L.A., Kojima N., Kittok R.J., Kinder J.E., 1993. Yearling bulls shorten the duration of postpartum anestrus in beef cows to the same extent as do mature bulls. J. Anim. Sci., 71, 306-309.

Dahl G.E., Buchanan B.A., Tucker H.A., 2000. Photoperiodic effects on dairy cattle: a review. J. Dairy Sci., 83, 885-893.

David I., Robert-Granié C., Manfredi E., Lagriffoul G., Bodin L., 2008. Environmental and genetic variation factors of artificial insemination success in French dairy sheep. Animal, 2, 979-986.

Delgadillo J.A., Leboeuf B., Chemineau P., 1993. Maintenance of sperm production in bucks during a third year of short photoperiodic cycles. Reprod. Nutr. Dev., 33, 609-617.

Delgadillo J.A., Carrillo E., Morán P., Duarte D., Chemineau P., Malpaux B., 2001. Induction of sexuel acrivity of male creole goats in subtropical northen Mexico using long days and melatonin. J. Anim. Sci., 79, 2245-2252.

Directive 98/58/CE du Conseil du 20 juillet 1998 concernant la protection des animaux dans les élevages. Journal Officiel des Communautés Européennes L 221 du 8 août 1998, 27.

Disenhaus C., 2008. La détection des chaleurs chez la vache laitière. BTIA, 128, 16-21.

Disenhaus C., Grimard B., Trou G., Delaby L., 2005. De la vache au système : s'adapter aux différents objectifs de reproduction en élevage laitier ? Renc. Rech. Rum., 125-136.

Dubbels R., Reiter R.J., Klenke E., Goeble A., Ehlers C., Schiwara H.W., Schloot W. 1995. Melatonin in edible plants identified by radioimmunoassay and by high performance liquid chromatography-mass spectrometry. J. Pineal Res. $18,28-31$

Fatet A., Leboeuf B., Fréret S., Druart X., Bodin L., Caillat H., David I., Palhière I., Boué P., Lagriffoul G., 2008. L'insémination dans les filières ovines et caprines. Renc. Rech. Rum. 355-358

Flores J.A. Véliz F.G., Pérez-Villanueva J.A., Martínez de la Escalera G., Chemineau P., Poindron P., Malpaux B., Delgadillo J.A., 2000. Male reproductive condition is the limiting factor of efficiency in the male effect during seasonal anoestrus in female goats. Biol. Reprod., 62 , 1409-1414.

France Bovins Croissance, 2007. Résultats du Contrôle des Performances bovins allaitants. 94p.

France Contrôle Laitier, 2007. Résultats de Contrôle Laitier des espèces bovine et caprine. $107 \mathrm{p}$.

France Génétique Elevage, 2007. Dispositif Génétique - Chiffres clés ruminants 2007, 8p

Garel J.P., Gauthier D., Petit M., Thimonier J. 1987. Influence of photoperiod on the postpartum changes in live weight and ovarian-function in suckled cows. Reprod. Nutr. Dev., 27, 305306.

Gérard O., Ponsart C., Petit M., Humblot P. 2008. Evolution des techniques de préparation et de mise en place de la semence en insémination artificielle chez les bovins. Renc. Rech. Rum., 351-354

Grimard B., Disenhaus C., 2005. Les anomalies de reprise de la cyclicité après vêlage. Le Point Vétérinaire, Reproduction des ruminants, Numéro Spécial, 36, 16-21.
Grimard B., Humblot P., Ponter A.A., Chastant S., Constant F., Mialot J.P., 2003. Efficacité des traitements de synchronisation des chaleurs chez les bovins. INRA Prod. Anim., 16, 211-227.

Hälli O., Tast A., Heinonen M., Munsterhjelm C., Valros A., Peltoniemi O.A.T., 2008. Short or long day light regimes may not affect reproductive performance in the sow. Reprod. Dom. Anim., 43, 708-712.

Hermansen J.E., 2005. Integration of organic pig production into land use. In: Organic pig production in free range systems. Sundrum A. Weissmann F. (Eds), Fal Agricultural Research. Special Issue, 3-12.

Hughes P.E., 1982. Factors affecting natural attainment of puberty in the gilt. In: Control of pig reproduction. Cole D.J.A., Foxcroft G.R. (Eds), Butterworths, London, UK, 161-177.

Hughes P.E., Thorogood K.L., 1999. A note on the effects of contact frequency and time of day of boar exposure on the efficacy of the boar effect. Anim. Reprod. Sci., 57, 121-124.

Hulten F., Wallenbeck A., Rydhmer L., 2006. Ovarian activity and oestrous signs among group-housed, lactating sows: influence of behaviour, environment and production. Reprod. Dom. Anim., 41, 448-454.

Humblot P., Grimard B., 1996. Endocrinologie du post-partum et facteurs influençant le rétablissement de l'activité ovarienne chez la vache. Le Point Vétérinaire, Reproduction des ruminants, Numéro Spécial, 28, 73-80.

IFIP-GTTT, 2008. Age des porcelets au sevrage et durée d'allaitement. Fiche technique IFIPAtout porc. http://www.ifip.asso.fr/eco/resultat/ pdf/atout/Fichesept08.pdf

Institut de l'élevage, Office de l'élevage, 2008. Les mises bas tardives, un nouvel itinéraire pour gérer la saisonnalité : incidences techniques et économiques. Collection résultats, février 2008, 39.

Kemp B., Soede N.M., Langendijk P., 2005. Effect of boar contact and housing condition on estrus expression in sows. Theriogenology, 62 , 643-656.

Kis R.K, Bilkei G., 2003. Effect of a phytogenic feed additive on weaning-to-estrus interval and farrowing rate in sows. J Swine Health Prod., 11, 296-299.

Lauritsen H.B., Sorensen G.S., Larsen V.A., 2000. Organic pig production in Denmark. In: Ecological Animal husbandry in the Nordic countries. Hermansen J.E., Lund, Vonne, Thuen, Erling, (Eds), DARCOF Report 2, Tjele, Denmark, 113-118.

Le Mezec P., Barbat A., 2007. Un répit dans la baisse de fertilité. BTIA, 126, 15-18.

López-Sebastian A., González-Bulnes A., Carrizosa J.A., Urrutia B., Díaz-Delfa C., Santiago-Moreno J., Gómez-Brunet A., 2007. New estrus synchronization and artificial insemination protocol for goats based on male exposure, progesterone and cloprostenol during the non-breeding season. Theriogenology, 68, 10811087.

Maton C., Montagnac D., Viudes G., Bouquet P., Bocquier F., 2008. Les applications de l'identification électronique des petits ruminants au service de l'élevage biologique. Innovations Agronomiques, 4, 67-71.

Monje A.R., Alberio R., Schiersmann G., Chedrese J., Carou N., Callejas S.S., 1992. Male 
effect on the post-partum sexual activity of cows maintained on two nutritional levels. Anim. Reprod. Sci., 29, 145-156.

Paterson A.M., Pearce G.P., Antuono M.F.D., 1991. Seasonal variation in attainment of puberty in isolated and boar-exposed domestic gilts. Ânim. Reprod. Sci., 24, 323-333.

Pavie J., Rétif R., 2006. Facteurs de variation des performances technico-économiques des exploitations d'élevage bovin en agriculture biologique. Renc. Rech. Rum., 373-376.

Pellicer-Rubio M.T., Leboeuf B., Bernelas D., Forgerit Y., Pougnard J.L., Bonné J.L., Senty, E., Chemineau P., 2007. Highly synchronous and fertile reproductive activity induced by the male effect during deep anoestrus in lactating goats subjected to treatment with artificially long days followed by a natural photoperiod. Anim. Reprod. Sci., 98, 241-258.

Pellicer-Rubio M.T., Leboeuf B., Bernelas D., Forgerit Y., Pougnard J.L., Bonnée J.L., Senty E., Breton S., Brun F., Chemineau P., 2008. High fertility using artificial insemination during deep anoestrus after induction and synchronisation of ovulatory activity by the «male effect» in lactating goats subjected to treatment with artificial long days and progestagens. Anim. Reprod. Sci., $109,172-188$.

Picard-Hagen N., Saint-Blancat M., Ponsart C. Ennuyer M., Defachelles J., DesCôteaux L., 2009. Comment utiliser les programmes de synchronisation de la reproduction chez la vache laitière en France. Le Nouveau Praticien Vétérinaire, Elevages et santé, 2, 377-386.

Ponsart C., Fréret S., Grimard B., Humblot P., Deletang F., Driancourt M.A., 2005. Du nouveau sur l'utilisation des progestagènes. BTIA, 117, 20-25.

Porc Performances, 2007. IFIP-Institut du Porc. Edition 2008, 43p.

Prunier A., Dourmad J.Y., Etienne M., 1994. Effect of light regimen under various ambient temperatures on sow and litter performance. J. Anim. Sci., 72, 1461-1466.

Règlement CEE n² 2092/91 du Conseil du 24 juin 1991 concernant le mode de production biologique de produits agricoles et sa présentation sur les produits agricoles et les denrées alimentaires. Journal Officiel de l'Union Européenne L 198 du 22 juillet 1991, 105.

Règlement CEE n ${ }^{\circ}$ 834/2007 du Conseil du 28 juin 2008 relatif à la production biologique et à l'étiquetage des produits biologiques et abrogeant le règlement CEE $n^{\circ}$ 2092/91. Journal Officiel de l'Union Européenne L 189 du 20 juillet 2007,23

Règlement CEE $\mathrm{n}^{\circ}$ 889/2008 de la Commission du 5 septembre 2008 portant modalités d'application du règlement $\mathrm{CE} \mathrm{n}^{\circ}$ $834 / 2007$ du Conseil relatif à la production biologique et à l'étiquetage des produits biologiques en ce qui concerne la production biologique, l'étiquetage et les contrôles. Journal Officiel de l'Union Européenne L 250 du 18 septembre 2008, 84

Restall B.J., 1988. The artificial insemination of Australian goats stimulated by the «buck effect». Proc. Aust. Soc. Anim. Prod., 17, 302-305.

Roberson M.S., Wolfe M.W., Stumpf T.T., Werth L.A., Cupp A.S., Kojima N., Wolfe P.L., Kittok R.J., Kinder J.E., 1991. Influence of growth rate and exposure to bulls on age at puberty in beef heifers. J. Anim. Sci., 69, 2092 2098.

Roy F., Maurel M.C., Combes B., Vaiman D., Cribiu E.P., Lantier I., Pobel T., Delétang F., Combarnous Y., Guillou F., 1999. The negative effect of repeated equine chorionic gonadotropin treatment on subsequent fertility in Alpine goats is due to a humoral immune response involving the major histocompatibility complex. Biol. Reprod., 60, 805-813.

Saint-Dizier M., 2005. La détection des chaleurs chez la vache. Le Point Vétérinaire,
Reproduction des ruminants, Numéro Spécial, $36,22-27$.

Scaramuzzi R.J., Martin G.B., 2008. The importance of interactions among nutrition, seasonality and socio-sexual factors in the development of hormone-free methods for controlling fertility. Reprod. Dom. Anim., 43, Suppl. 2, 129136.

Serieys F., 2007. Photopériode : un paramètre influant et influençable. Le Point Vétérinaire, 279, 29-32.

Telefo P.B., Moundipa F.P., Tchouanguep M.F., Tchana A., 1998. Effect of aqueous extract of Aloe buettneri, Justicia insularis, Hibiscus macranthus, Dicliptera verticillata on some physiological and biochemical parameters of reproduction in immature female rats. Etnopharmacology, 63, 193-200.

Thimonier J., Cognié Y., Lassoued N., Khald G., 2000. L'effet mâle chez les ovins : une technique actuelle de maîtrise de la reproduction. INRA Prod. Anim., 13, 223-231.

Tournadre H., Bocquier F., Petit M. Thimonier J., Benoit M., 2002. Efficacité de l'effet bélier chez la brebis limousine à différents moments de l'anoestrus saisonnier et selon la durée de l'intervalle tarissement-mise en lutte. Renc. Rech. Rum., 9, 143-146.

Tournadre H., Pellicer-Rubio M.T., Bocquier F., 2008. Maîtriser la reproduction en élevage ovin biologique : influence de facteurs d'élevage sur l'efficacité de l'effet bélier. Innovations Agronomiques, 4, 85-90.

Veysset P., Becherel F., Glouton J., 2006. Elevage bovin allaitant biologique du Massif Central : analyse des résultats technicoéconomiques. Renc. Rech. Rum., 378.

Walkden-Brown S.W., Martin G.B., Restall B.J., 1999. Role of male-female interaction in regulating reproduction in sheep and goats. $\mathrm{J}$. Reprod. Fert., Suppl. 52, 243-257.

\section{Résumé}

La maîtrise de la reproduction contribue à optimiser la productivité de l'exploitation. La réglementation de la production biologique n'impose pas de conduite de reproduction spécifique, mais limite les pratiques autorisées parmi l'ensemble des stratégies disponibles pour les éleveurs. Les conséquences de ces limitations sont plus ou moins pénalisantes selon l'espèce animale considérée. Ainsi, l'interdiction des traitements hormonaux représente le principal obstacle pour la maîtrise de la reproduction en agriculture biologique (AB) chez les petits ruminants et les porcins.

La maîtrise de la saisonnalité de la reproduction est un objectif pour la plupart des éleveurs ovins et caprins. Dans ces deux espèces, la reproduction est possible tout au long de l'anoestrus saisonnier grâce à des traitements lumineux sans recours à la mélatonine, mais des difficultés de mise en ouvre font qu'ils sont aujourd'hui peu utilisés en AB.

En élevage conventionnel ovin et caprin, l'insémination artificielle (IA) est réalisée à un moment fixe après synchronisation hormonale des ovulations et sans détection d'oestrus. En AB, l’IA est autorisée sur chaleurs naturelles : cela implique la pratique systématique de la détection des chaleurs sur plusieurs jours pour identifier les femelles à inséminer. Pour cette raison, l'IA est très peu pratiquée en $A B$ chez ces deux espèces. La synchronisation des ovulations par effet mâle est une alternative prometteuse pour simplifier les protocoles d'IA, facilitant l'accès des éleveurs $\mathrm{AB}$ aux schémas de sélection et donc la généralisation de cette pratique.

La conduite de reproduction la plus courante en élevage de porc conventionnel est dite en 7 bandes. La durée du cycle d'une bande est de 21 semaines dont 4 semaines d'allaitement. $\mathrm{En} \mathrm{AB}$, le règlement européen impose l'augmentation du temps d'allaitement des porcelets à $40 \mathrm{j}$ minimum. En pratique, le sevrage a lieu à $42 \mathrm{j}$ avec une conduite en 8 bandes, le cycle de reproduction s'étend donc à 23 semaines.

En AB comme en conventionnel, le sevrage est la principale méthode utilisée pour la synchronisation de l'oestrus des truies dans leur bande. L'efficacité de la conduite en bandes est toutefois pénalisée en AB par l'interdiction des traitements hormonaux, utilisés fréquemment en conventionnel pour intégrer les cochettes dans les bandes, pour recycler des femelles décalées ou pour prévenir des oestrus de lactation. Cependant, l'interdiction de l'utilisation d'hormones ne pénalise pas la pratique de l'IA car elle est réalisée sur oestrus naturels synchronisés par le sevrage chez la majorité des producteurs en $\mathrm{AB}$ et en élevage conventionnel. 
Chez les bovins, l'insémination est pratiquée de manière générale sur chaleurs naturelles. Lors de la mise à la reproduction, la mise en place de la semence est réalisée par insémination artificielle (majoritaire en élevage laitier) ou par monte naturelle (majoritaire en élevage allaitant). L'interdiction des traitements hormonaux d'induction et de synchronisation des chaleurs n'est donc pas pénalisante en AB par rapport à l'élevage conventionnel. La mise à la reproduction est possible toute l'année, avec des contraintes qui diffèrent selon les objectifs de production.

Les voies de recherche pour le développement de méthodes naturelles de maîtrise de la reproduction utilisables en $A B$ sont très diverses, multidisciplinaires et souvent communes aux différentes espèces de mammifères d'élevage. L'INRA contribue fortement à l'amélioration des méthodes existantes et à leur adaptation aux contraintes d'élevage. Il s'implique également dans la conception et la validation de nouvelles approches de maîtrise de la reproduction sans hormones. Ce développement ne pourra connaître un véritable essor que dans le cadre d'une relation étroite entre la recherche finalisée et les professionnels.

\section{Abstract}

\section{Available methods for the control of reproduction in domestic mammals and their interest for organic animal production}

The control of reproduction is necessary to optimise the productivity of livestock production systems. The regulations of organic farming do not impose specific reproductive practices among those that are already in use except that some of them are not authorized. Whatever the animal species, the success of reproduction in organic systems mainly relies on the choice of adapted breeds.

Because sheep and goats are well-known seasonal breeders the major issue is to overcome this limitation of reproductive possibilities. In these organic farming systems the reproduction is possible all year round thanks to the manipulation of daylight duration without using melatonin, however up to now these treatments are not developed. The male effect is another method to overcome seasonal blockade and that allows synchronizing lambing and kidding without any hormonal treatments. This method is however rather difficult to apply in all situations. Artificial insemination (AI) is the main way to benefit from selection's progress. In conventional systems AI is performed at fixed time after hormonal synchronization of ovulations without necessity to detect oestrus. AI, which is authorized on natural oestrus, is not really used in organic flocks because they are spread over several days. Synchronization of oestrus with the male effect is however a promising way to perform AI and allow organic breeders to benefit from selection schemes.

In conventional swine production systems the classical practice is to drive sows into a 7 batches system at 3 weeks interval. The productive cycle duration is of 21 weeks including 28 days of suckling. In the European regulation of organic farming systems, the piglet suckling period should not be shorter than 40 days. Practically the weaning will occur at 42 days with a 8 batches system. Hence the whole productive cycle of the sow will be lengthened up to 23 weeks. In organic system, like in conventional systems, weaning is the main method to synchronize sows oestrus within a given batch. The overall efficiency of organic production is however reduced because no hormonal treatment is allowed either to synchronize gilts or to transfer open sows into another band and those that may show oestrus during lactation. In all pig production systems AI is performed after oestrus detection.

In cattle, $\mathrm{AI}$ is generally performed after detection of natural oestrus. Reproduction is mainly performed through $\mathrm{AI}$ in dairy cows and with bull mating in beef cows. Thus the fact that hormonal treatments are not allowed in organic systems do not penalise them. Furthermore reproduction is possible all year round except that it is performed according to productive goals and local constraints.

The development of natural methods for the control of reproduction in organic livestock systems implies numerous research approaches and multidisciplinary programs, often common to different species. INRA is strongly involved in the study of hormone-free methods and their adaptation to breeding constraints.

PELLICER-RUBIO M.-T., FERCHAUD S., FRERET S., TOURNADRE H., FATET A., BOULOT S., PAVIE J., LEBOEUF B., BOCQUIER F., 2009. Les méthodes de maîtrise de la reproduction disponibles chez les mammifères d'élevage et leur intérêt en agriculture biologique. Inra Prod. Anim., 22, 255-270. 